\title{
Towards viable cosmological models of disformal theories of gravity
}

\author{
Jeremy Sakstein \\ Department of Applied Mathematics and Theoretical Physics, Centre for Mathematical Sciences, \\ Cambridge CB3 OWA, United Kingdom; Institute of Cosmology and Gravitation, University of Portsmouth, \\ Portsmouth PO1 3FX, United Kingdom; and Perimeter Institute for Theoretical Physics, \\ 31 Caroline Street North, Waterloo, Ontario N2L 6B9, Canada \\ (Received 29 September 2014; published 26 January 2015)
}

\begin{abstract}
The late-time cosmological dynamics of disformal gravity are investigated using dynamical systems methods. It is shown that in the general case there are no stable attractors that screen fifth forces locally and simultaneously describe a dark energy dominated universe. Viable scenarios have late-time properties that are independent of the disformal parameters and are identical to the equivalent conformal quintessence model. Our analysis reveals that configurations where the Jordan frame metric becomes singular are only reached in the infinite future, thus explaining the natural pathology resistance observed numerically by several previous works. The viability of models where this can happen is discussed in terms of both the cosmological dynamics and local phenomena. We identify a special parameter tuning such that there is a new fixed point that can match the presently observed dark energy density and equation of state. This model is unviable when the scalar couples to the visible sector but may provide a good candidate model for theories where only dark matter is disformally coupled.
\end{abstract}

DOI: $10.1103 /$ PhysRevD.91.024036

PACS numbers: 04.50.Kd, 95.36.+x, 98.80.-k

\section{INTRODUCTION}

The observation of the acceleration of the cosmic expansion [1,2] has prompted a renewed interest in modified theories of gravity as a potential driving mechanism. Amongst the plethora of candidate theories (see [3] for a review), those that contain screening mechanisms (see [4] for a review) have been particularly well studied due to their ability to hide the additional or fifth forces on solar system scales. Well-studied nonlinear screening mechanisms-meaning that the local dynamics act to suppress fifth forces-include the chameleon mechanism $[5,6]$ and similar $[7,8]$ as well as the Vainshtein mechanism [9]. These theories can be described by a conformal coupling of a scalar to matter through the metric [10]. Theories that contain a disformal coupling to matter have been studied in the context of dark matter and dark energy [12-21] (see also [22] for an application to inflation) but unlike chameleons, which have been well studied and constrained on small scales [23-28], the local behavior of disformal theories has been relatively unstudied (although see $[29,30]$ for tests of Tensor-Vector-Scalar theory and a model that satisfies the Cassini bound on light bending by the Sun).

Recently, [21] has performed a thorough investigation into the local dynamics of the scalar. Nonrelativistic objects inside a Friedmann-Robertson-Walker (FRW) universe will source a field profile such that the fifth force is $F_{5}=2 Q^{2} F_{\mathrm{N}}-F_{\mathrm{N}}$ is the Newtonian force-where the local scalar charge $Q$ depends on the conformal coupling, the disformal parameters and the first and second time derivatives of the cosmological field. There, it was argued that there are no nonlinear screening mechanisms beyond those mentioned above. It was shown, however, that when the conformal coupling is absent [31] these theories screen linearly - by which we mean the local scalar charge is suppressed on all scales through the cosmological dynamics and not the local ones-whenever the cosmological dynamics are such that the field is slowly rolling.

The aim of this work is to investigate more general models where the conformal factor is nonzero and see whether one can find models that can drive the cosmic acceleration at late times while simultaneously suppressing the local scalar charge. We will do this using dynamical systems techniques. These are powerful tools to classify the late-time behavior of nonlinear systems despite the lack of analytic solutions. Their power lies in their ability to classify all solutions of the system in terms of a few fixed points independently of the initial conditions. Without them, one would be reduced to solving the problem numerically for all possible parameters and initial conditions, a problem that is clearly intractable. Since the relevant cosmological parameters have well-defined values at these points, one can know the final state the Universe will evolve to. Dynamical systems methods have previously been used in the study of dark energy models in the form of quintessence [33] and conformally coupled theories [34,35] (see [36] for a review). There, they find dark energy dominated solutions by looking for points where the density parameter and equation of state is close to the observed values [37]. Here, we will do the same for the disformal system, examining the local scalar charge as well in order to classify the linear screening. Unlike the simple cases mentioned above, the disformal system requires use of the center manifold technique, which is necessary whenever different directions in phase space 
evolve on different time scales. Interestingly, we will see that some of the attractors of the conformal system are saddle points of the disformal system and the evolution can be very different at late times.

These theories have potential instabilities since the determinant of the Jordan frame metric can become singular. Several authors $[14,15,17]$ have observed numerically that solutions tend to slow down to avoid this and have dubbed this phenomenon a natural resistance to pathology. Using the techniques described above, we will show that any singularity is only reached in an infinite amount of time, thereby explaining this phenomenon. Whether or not this is a pathology of the theory has been debated and here we will argue that whenever this singularity is present, the nonrelativistic limit is not well defined and one generally expects large fifth forces and time variations in Newton's constant as the singularity is approached.

Since portions of this paper are technical, we state our main results unambiguously below.

(i) The phase space is three dimensional, in contrast to the purely conformal case. In the general case, there is one new dark energy dominated solution (discussed in the next bullet point) and one retains all of the solutions found in the purely conformal case. The dark energy dominated points found also in the conformal case do not screen linearly unless the conformal factor is absent.

(ii) There are parameter choices where the purely conformal fixed points are saddle points. In this case, the system ultimately evolves towards a dark energy dominated solution where the determinant of the Jordan frame metric is singular and there are large unscreened fifth forces.

(iii) There is a special tuning in parameter space where the dimension of the phase space is reduced to two. In this case there is a new stable attractor where one can reproduce the present dark energy density parameter and equation of state by making an appropriate choice for the model parameters. Unfortunately, the metric singularity is approached along this attractor and hence the model is not viable.

(iv) The conclusions drawn here assume that the disformal coupling of the scalar to matter is universal. If one were to relax this and only couple the scalar to the dark sector (i.e. visible matter couples only to the Einstein frame metric) then many of the solutions claimed to be unviable here may be tenable. This requires further investigation and we discuss this in detail in the conclusions.

This paper is organized as follows: The theory is introduced in Sec. II where we discuss the local fifth forces, the metric singularity and the potential problems it entails. Section III constitutes the main body of this work. There it is shown that there are no stable dark energy dominated solutions that simultaneously have zero local scalar charge. Furthermore, we show that the singularity in the Jordan frame metric is only reached in the infinite future. In Sec. IV we examine the special parameter choices such that the phase space is two dimensional and find a new fixed point that can reproduce the observed dark energy parameters but has a metric singularity in the infinite future. We conclude in Sec. V. A brief introduction to dynamical systems including center manifold techniques is provided in Appendix A for the unfamiliar reader.

\section{DISFORMAL THEORIES OF GRAVITY}

Disformal theories of gravity are described by the following action:

$$
S=M_{\mathrm{pl}^{2}}^{2} \int \mathrm{d}^{4} x \sqrt{-g}\left[\frac{R}{2}+X-V(\phi)\right]+S_{\mathrm{m}}\left[\tilde{g} ; \Psi_{\mathrm{i}}\right],
$$

where the various matter fields $\Psi_{\mathrm{i}}$ are coupled to the Jordan frame metric

$$
\tilde{g}_{\mu \nu}=A^{2}(\phi)\left(g_{\mu \nu}+\frac{B^{2}(\phi)}{\Lambda^{2}} \partial_{\mu} \phi \partial_{\nu} \phi\right)
$$

and $X \equiv-1 / 2 \nabla_{\mu} \phi \nabla^{\mu} \phi . g_{\mu \nu}$ is the Einstein frame metric. $A$ and $B$ are known as the conformal and disformal factors respectively and we define the following quantities [38]

$$
\alpha(\phi) \equiv \frac{\mathrm{d} \ln A(\phi)}{\mathrm{d} \phi} \quad \text { and } \quad \gamma(\phi) \equiv \frac{\mathrm{d} \ln B(\phi)}{\mathrm{d} \phi} .
$$

Varying the action (1) with respect to $\phi$, one finds the equation of motion for the field

$$
\square \phi=V(\phi)_{, \phi}+Q,
$$

where

$$
\begin{aligned}
Q \equiv & \nabla_{\mu}\left(\frac{B^{2}(\phi)}{\Lambda^{2}} T_{\mathrm{m}}{ }^{\mu \nu} \nabla_{\mu} \phi\right)-\alpha(\phi) T_{\mathrm{m}} \\
& -\frac{B(\phi)^{2}}{\Lambda^{2}}[\gamma(\phi)+\alpha(\phi)] T_{\mathrm{m}}{ }^{\mu \nu} \nabla_{\mu} \phi \nabla_{\nu} \phi .
\end{aligned}
$$

Here $T_{m}^{\mu \nu}=2 / \sqrt{-g} \delta S_{\mathrm{m}} / \delta g_{\mu \nu}$ is the energy-momentum tensor for matter. Since the scalar is coupled directly to matter, this is not conserved [39] and one instead has

$$
\nabla_{\mu} T_{\mathrm{m}}{ }^{\mu \nu}=-Q \nabla^{\nu} \phi
$$

Contracting this with $\nabla_{\nu} \phi$, one can algebraically solve for $\nabla_{\mu} T^{\mu \nu} \nabla_{\nu} \phi$, which can be used to eliminate all derivatives of the energy-momentum tensor in (5) so that (4) becomes 


$$
\begin{aligned}
(1 & \left.-\frac{2 B^{2} X}{\Lambda^{2}}\right) \square \phi-8 \pi G \frac{B^{2}}{\Lambda^{2}} T_{\mathrm{m}}^{\mu \nu} \nabla_{\mu} \nabla_{\nu} \phi \\
& =-8 \pi \alpha G T_{\mathrm{m}}-8 \pi G \frac{B^{2}}{\Lambda^{2}}(\alpha-\gamma) T_{\mathrm{m}}^{\mu \nu} \partial_{\mu} \phi \partial_{\nu} \phi+\chi V(\phi)_{\phi} .
\end{aligned}
$$

In practice, this equation is the more useful of the two to work with and here we will do so whenever possible.

The determinants of the two metrics are related by (see [17], Appendix A)

$$
\frac{\sqrt{-\tilde{g}}}{\sqrt{-g}}=A^{4} \sqrt{1-\frac{2 B^{2} X}{\Lambda^{2}}} .
$$

Note that it is possible for the right-hand side to become zero so that the metric is singular, which is a potential problem for the theory. Cosmologically, several authors $[14,15,17]$ have numerically found a natural resistance to pathology in the solutions where the cosmological time evolution of the field slows down at late times and the singularity is avoided. We will address this issue later and show that certain models can evolve towards the metric singularity but only in an infinite time, thereby explaining the numerical observations of this pathology resistance.

\section{A. Local behavior and pathologies}

In [21], it was shown that when expanded around a FRW background, the fifth force arising in these theories is

$$
F_{5}=2 Q^{2} F_{\mathrm{N}}
$$

where $F_{\mathrm{N}}$ is the Newtonian force and the local scalar charge $Q$ is

$$
Q \equiv \frac{\alpha+\frac{B^{2}}{\Lambda^{2}}\left(\ddot{\phi}_{\infty}+\dot{\phi}_{\infty}^{2}[\gamma-\alpha]\right)}{1-\frac{B^{2} \dot{\phi}_{\infty}^{2}}{\Lambda^{2}}},
$$

where $\phi_{\infty}$ is the cosmological (homogeneous) component of the field and an overdot denotes a derivative with respect to coordinate time $t$ in the Einstein frame. For completeness, we briefly present the derivation of this formula here. Using the Einstein frame coordinates $\mathrm{d} s^{2}=-(1+2 \Phi) \mathrm{d} t^{2}+$ $(1-2 \Psi) \delta_{i j} \mathrm{~d} x^{i} \mathrm{~d} x^{j}$ we expand the field equations about the cosmological background value so that $\phi-\phi_{\infty}(t)+$ $\varphi(r, t)$ and ignore all terms that are post-Newtonian [40]. With these assumptions, Eq. (7) becomes

$$
\nabla^{2} \varphi=8 \pi Q G \rho .
$$

This is nothing but the Poisson equation with $G \rightarrow 2 Q G$ so that $\varphi=2 Q \Phi_{\mathrm{N}}$, where $\Phi_{\mathrm{N}}$ is the Newtonian potential. Next, we need the fifth force, which can be found as follows. Defining the tensor

$$
\mathcal{K}_{\mu \nu}^{\alpha} \equiv \tilde{\Gamma}_{\mu \nu}^{\alpha}-\Gamma_{\mu \nu}^{\alpha},
$$

the geodesic equation can be written (note that matter moves on geodesics of $\tilde{g}_{\mu \nu}$ since this is the metric that couples to matter) in terms of purely Einstein frame quantities. In the nonrelativistic limit one has $\mathrm{d} x^{0} / \mathrm{d} \tau \gg$ $\mathrm{d} x^{i} / \mathrm{d} \tau-\tau$ is the proper time in the Jordan frame-so that

$$
\ddot{x}^{i}+\Gamma_{00}^{i}=-\mathcal{K}_{00}^{i} .
$$

In this form, one can use the familiar result $\Gamma_{00}^{i}=\partial^{i} \Phi_{\mathrm{N}}$ [41] to see that all of the effects of the fifth force are contained within $\mathcal{K}_{00}^{i}$. Using Eq. (2), one finds that $\mathcal{K}_{00}^{i}=$ $Q \partial^{i} \varphi$ (see [21]) so that the fifth force is given by $F_{5}=2 Q^{2} F_{\mathrm{N}}$, where $F_{\mathrm{N}}$ is the Newtonian force. The theory then behaves as one with a time-varying gravitational constant $G_{\text {eff }}=G\left(1+2 Q^{2}\right)$ when $\dot{\phi}_{\infty} \ll \Lambda$ (see the discussion below).

In [21], we examined models where $\alpha(\phi)=0$ and argued that any model where the scalar potential has a minimum can naturally screen fifth forces because at late times, when the field is slowly rolling, $Q \approx 0$. In this work, we wish to address the more general question: can we find models with $\alpha \neq 0$ where the scalar charge is identically zero at late times and the Universe is dark energy dominated? In order to answer this, one must use dynamical systems techniques since this allows one to explore the entire solution space of the theory without numerically solving each model with every possible set of initial conditions.

One important question to address is whether solutions that evolve towards a singularity in the infinite future should be considered viable or not. In this subsection we will argue that they should not, at least when the scalar couples to the visible sector.

Note that the denominator in (10) is zero precisely when the metric singularity occurs. The simplest interpretation of this is that the fifth force becomes infinite whenever the numerator does not tend to zero at the same rate. In this case one would denounce these models as unviable unless the numerator approaches zero. Such a property cannot be a generic feature of any given model. We will see below that the phase space of cosmological solutions is three dimensional and so the lack of a multidimensional analogue of l'Hôpital's rule means that the value of $Q$ is not set by the properties of the fixed point. Instead, its value depends on the trajectory through phase space along which the point is approached. For this reason, a solution where fifth forces are screened at late times using this method is only found using a special tuning of both the model parameters and the initial conditions.

The discussion above is predicated on the assumptions that the nonrelativistic limit derived in [21] is valid when $B \dot{\phi}_{\infty} \rightarrow \Lambda$ and that one can use the Einstein frame coordinates to describe the physics of local observers. 
In fact, when this is the case the nonrelativistic limit is far more subtle and may not even exist. To see this, consider the case where $A(\phi)=B(\phi)=1$ [42]. If one chooses coordinates $\left\{t, x^{i}\right\}$ such that the Einstein frame metric is FRW, i.e. $g_{\mu \nu}=\operatorname{diag}\left(-1, a_{\mathrm{E}}^{2}(t), a_{\mathrm{E}}^{2}(t), a_{\mathrm{E}}^{2}(t)\right)$ and the homogeneous component of the scalar is $\phi_{\infty}(t)$ then using Eq. (2) one finds that the line element in the Jordan frame is

$$
\mathrm{d} \tilde{s}=-\mathcal{N}^{2} \mathrm{~d} t^{2}+a_{\mathrm{E}}^{2}(t) \delta_{i j} \mathrm{~d} x^{i} \mathrm{~d} x^{j},
$$

where the Jordan frame lapse is

$$
\mathcal{N}^{2} \equiv 1-\frac{\dot{\phi}_{\infty}^{2}}{\Lambda^{2}}
$$

One can immediately see the Jordan frame manifestation of the metric singularity: the lapse becomes zero. When $\dot{\phi}_{\infty} \sim \Lambda$ the proper time for stationary observers in the Jordan frame is not given by $t$ but rather by $T$, where

$$
\mathrm{d} T=\mathcal{N} \mathrm{d} t .
$$

Since it is the Jordan frame metric that couples directly to matter, it is this metric that one must use to calculate the observables in this theory. Indeed, the nonrelativistic limit was derived in [21] by transforming the nonrelativistic limit of the geodesic equation to the Einstein frame in order to allow an interpretation of the new effects as a fifth force. This derivation assumes a locally inertial reference frame, which requires one to work with $T$ and not $t$. Thus, when $\dot{\phi}_{\infty} \ll \Lambda$ this is perfectly valid and Eq. (9) describes a fifth force due to a field with scalar charge

$$
Q \approx \alpha+\frac{B^{2}}{\Lambda^{2}}\left(\ddot{\phi}_{\infty}+\dot{\phi}_{\infty}^{2}[\gamma-\alpha]\right) .
$$

When the converse is true, the nonrelativistic limit, if it exists, must be found using $T$ and not $t$. Defining a nonrelativistic limit in this limit is difficult because one actually has two speeds in the problem: the speed of light, $c_{\text {light }}=c$, and the speed of tensor propagation in the Jordan frame (see [43])

$$
c_{\text {tensors }}^{2}=1-\frac{\dot{\phi}_{\infty}^{2}}{\Lambda^{2}} .
$$

Note that one can instead interpret this as a varying speed of light in the Einstein frame [44-46], although one must be careful to interpret this speed correctly, especially when different species couple to different metrics. It is not clear whether a consistent nonrelativistic limit exists when these differ greatly since one must choose a small parameter in order to expand the equations. This is typically $v / c$ where $v$ is a typical speed scale in the problem but a priori one does not know whether this should be $v / c_{\text {light }}, v / c_{\text {tensors }}$ or some combination of the two. Since the speed of tensors becomes very low when $\dot{\phi}_{\infty} \sim \Lambda$ one may worry that there is no nonrelativistic limit —or rather, that it is valid at speeds far smaller than those present in the Solar System-since one of the speeds in the problem is very low. A full analysis of this would require a complete self-consistent solution of the field equations in the Jordan frame to determine which, if any, approximations can be made. This is clearly well beyond the scope of this work.

Other potential issues that arise when $\dot{\phi}_{\infty} \sim \Lambda$ can be seen examining the Jordan frame action, which contains a term of the form (this can be found using the method of [47]):

$$
S \supset \int \mathrm{d}^{4} x \sqrt{-\tilde{g}} \frac{R}{16 \pi G} \sqrt{1-\frac{\tilde{\nabla}_{\mu} \phi \tilde{\nabla}^{\mu} \phi}{\Lambda^{2}}},
$$

where

$$
\tilde{\nabla}_{\mu} \phi \tilde{\nabla}^{\mu} \phi=\tilde{g}^{\mu \nu} \partial_{\mu} \phi \partial_{\nu} \phi
$$

Using the coordinate system defined by (16), one finds

$$
\frac{\mathrm{d} \phi_{\infty}}{\mathrm{d} T}=\frac{\dot{\phi}_{\infty}}{\mathcal{N}}
$$

and

$$
\mathrm{d} \tilde{s}=-\mathrm{d} T^{2}+a_{\mathrm{J}}^{2}(T) \delta_{i j} \mathrm{~d} x^{i} \mathrm{~d} x^{j},
$$

where the Jordan frame scale factor is $a_{\mathrm{J}}(T)=a_{\mathrm{E}}(t(T))$. From this, one can see that the effective value of Newton's constant in the Solar System is

$$
G_{\mathrm{N}}(t)=G \sqrt{1-\frac{\dot{\phi}_{\infty}^{2}}{\Lambda^{2}}}\left[1+f\left(\partial_{i} \varphi\right)\right],
$$

where $\phi=\phi_{\infty}+\varphi$ and $f\left(\partial_{i} \varphi\right)$ represents the effects of the inhomogeneous component of the field sourced by small-scale sources. One can see that theories where $\dot{\phi}_{\infty}$ approaches $\Lambda$ predict lower values of $G_{\mathrm{N}}$ in the Solar System than would be inferred using the Friedmann equation [this is similar to conformal theories where $G_{\mathrm{N}}=A^{2}\left(\phi_{\infty}\right) G$ [48]. This behavior is also seen in other theories that include nonminimal couplings to curvature tensors [49].

Finally, note that knowledge of the Einstein frame dynamics is not sufficient to determine the late-time dynamics in the Jordan frame when the solution approaches the metric singularity. To see this, note that

$$
H_{\mathrm{J}}=\frac{H_{\mathrm{E}}}{\mathcal{N}} \quad \text { and } \quad \frac{1}{a_{\mathrm{J}}} \frac{\mathrm{d}^{2} a_{\mathrm{J}}}{\mathrm{d} T^{2}}=\frac{1}{\mathcal{N}^{2}}\left(\frac{\ddot{a}_{\mathrm{E}}}{a_{\mathrm{E}}}+H_{\mathrm{E}} \frac{\ddot{\phi}_{\infty}}{\Lambda^{2}}\right) .
$$


At late times, we expect $H_{\mathrm{E}} \rightarrow 0$ but since $\mathcal{N} \rightarrow 0$ in the same limit the behavior of $H_{\mathrm{J}}$ cannot be determined using dynamical systems methods [50]. As far as the acceleration of the Universe is concerned, one expects the Einstein frame Hubble parameter to approach zero at late times and so an Einstein frame analysis is sufficient to determine whether or not $\ddot{a}_{\mathrm{J}}>0$, which is the true criterion for acceleration [51].

It is for the reasons presented in this subsection that we will consider models that approach the metric singularity as unviable in this work. We will discuss possible caveats and resolutions in the conclusions as well as possible future directions one could pursue in order to address these issues further

\section{B. Cosmology}

From here on we will work exclusively in the Einstein frame and we hence drop any subscripts on cosmological quantities such as $H$ and $a$. All quantities are given in the Einstein frame unless explicitly stated otherwise. The background cosmology (in coordinate time) is determined by the Friedmann equations

$$
\begin{aligned}
3 H^{2} & =8 \pi G \rho_{\mathrm{m}}+\frac{\dot{\phi}_{\infty}^{2}}{2}+V\left(\phi_{\infty}\right), \\
\dot{H} & =-4 \pi G \rho_{\mathrm{m}}-\frac{\dot{\phi}_{\infty}^{2}}{2}
\end{aligned}
$$

coupled to the equation of motion for the scalar and the matter density:

$$
\begin{gathered}
\ddot{\phi}_{\infty}+3 H \dot{\phi}_{\infty}+V\left(\phi_{\infty}\right)_{, \phi}=-Q_{0} \quad \text { and } \\
\dot{\rho}_{\mathrm{m}}+3 H \rho_{\mathrm{m}}=Q_{0} \dot{\phi}_{\infty}
\end{gathered}
$$

where

$$
Q_{0}=8 \pi G \rho_{\mathrm{m}} \frac{\alpha+\frac{B^{2}}{\Lambda^{2}}\left([\gamma-\alpha] \dot{\phi}_{\infty}^{2}-3 H \dot{\phi}_{\infty}-V_{, \phi}\right)}{1+\frac{B^{2}}{\Lambda^{2}}\left(8 \pi G \rho_{\mathrm{m}}-\dot{\phi}_{\infty}^{2}\right)},
$$

and $\rho=-T_{\mathrm{m}}$ with $T_{\mathrm{m}}$ the trace of the Einstein frame energy-momentum tensor. Note that since the scalar is nonminimally coupled to matter the energy-momentum tensor is not covariantly conserved in this frame, which leads to the modified continuity equation (28). We do not include radiation or other particle species in this work since we are interested in the late-time behavior of the system where all of these components are subdominant.

\section{DYNAMICAL SYSTEMS ANALYSIS}

\section{A. Phase space construction}

Dynamical systems have been used in cosmology (see [36] and references therein) to examine the late-time behavior of quintessence [33] and conformally coupled dark energy [35]. Here, we will extend the formalism to disformally coupled dark energy [52]. A brief introduction to the general techniques is given in Appendix A for the unfamiliar reader. We begin by introducing the new variables

$$
\begin{array}{ll}
x \equiv \frac{\phi_{\infty}^{\prime}}{\sqrt{6}}, & y \equiv \frac{\sqrt{V}}{\sqrt{3} H}, \\
\lambda \equiv-\frac{V_{, \phi}}{V}, & z \equiv \frac{B H}{\Lambda},
\end{array}
$$

where we have changed from coordinate time $t$ to $N \equiv$ $\ln a(t)$ and use a prime to denote derivatives with respect to $N$. In order to focus on the simplest case, we take $\lambda, \alpha$ and $\gamma$ to be constant so that

$$
V(\phi)=m_{0}^{2} e^{-\lambda \phi}, \quad A(\phi)=e^{\alpha \phi} \quad \text { and } \quad B(\phi)=e^{\gamma \phi},
$$

where $m_{0}$ is a mass scale associated with the scalar potential. Note that this is the coupled dark energy model of [35] extended to include an exponential disformal coupling. Such a model has previously been studied by [17] with $\alpha=0$ and so this is a generalization of their model to arbitrary conformal couplings. One should note that whereas this model makes a specific choice for the functional forms of the free functions, one can think of the fixed points found using this system as instantaneous fixed points for more complicated models [53]. Written in these variables, the Friedmann-scalar field system can be written in first-order autonomous form:

$$
\begin{aligned}
\frac{\mathrm{d} x}{\mathrm{~d} N}= & -3 x+\frac{3}{2} x\left(1+x^{2}-y^{2}\right)+\sqrt{\frac{3}{2}} y^{2} \lambda \\
& +\frac{\sqrt{\frac{3}{2}}\left(x^{2}+y^{2}-1\right)\left(\alpha+3 z^{2}\left(2 x^{2}(\gamma-\alpha)-\sqrt{6} x+y^{2} \lambda\right)\right)}{1+3\left(1-3 x^{2}-y^{2}\right) z^{2}},
\end{aligned}
$$

$\frac{\mathrm{d} y}{\mathrm{~d} N}=\frac{3}{2} y\left(1+x^{2}-y^{2}\right)-\sqrt{\frac{3}{2}} x y \lambda \quad$ and

$\frac{\mathrm{d} z}{\mathrm{~d} N}=\sqrt{6} \gamma x z-\frac{3}{2} z\left(x^{2}-y^{2}+1\right)$.

These are to be supplemented with the Friedmann constraint

$$
x^{2}+y^{2}+\Omega_{\mathrm{m}}=1,
$$

where $\Omega_{\mathrm{m}} \equiv 8 \pi G \rho_{\mathrm{m}} / 3 H^{2}$. This system is then three dimensional. In terms of the variables $\left\{\phi, a(t), \Omega_{\mathrm{m}}\right\}$ we have a first-order equation for $\Omega_{\mathrm{m}}$ and second-order 
equations for $\phi$ and $a(t)$ implying that we have a fivedimensional phase space. One can see that there is a symmetry of the solutions whereby we can rescale $H$ and the various other factors $\left(B, V\right.$ and $\left.\dot{\phi}_{\infty}\right)$ and leave the equations invariant. For this reason, one can scale $H$ out of the problem and work with the five quantities $\left\{x, y, z, \Omega_{\mathrm{m}}, a\right\}$. Equation (35) is a constraint equation and so we can use it to eliminate $\Omega_{\mathrm{m}}$ which reduces the dimension of the phase space to four. Finally, the phase space is reduced to three by the choice of time coordinate $N$. Since the equations are time-translation invariant in this coordinate, this allows us to remove all dependence on $a$ in the equations so that $x, y$ and $z$ are the only dynamical degrees of freedom and thereby reducing the dimension of the phase space to three. This leaves us with three coupled first-order equations including the constants $\{\lambda, \alpha, \gamma\}$. This is to be contrasted with the conformally coupled case where the phase space is two dimensional. This is because choosing $\lambda$ and $\alpha$ to be constant removes any dependence on $\phi$ in the equations for $x$ and $y$ whereas $B(\phi)$ remains in $Q_{0}$ even when $\gamma$ is constant. Note also that $\gamma=0$ does not reduce the dimensionality of the phase space because in this case $z$ depends on $H$, which cannot be eliminated in terms of $x$ and $y$ only and so the case where $B$ is constant is included in this analysis. On the other hand, when $\gamma=\lambda / 2$ we have the relation

$$
z y=\frac{m_{0}}{\sqrt{3} \Lambda}
$$

and so one may eliminate $z$ in terms of $y$ or vice versa. Therefore, $\gamma=\lambda / 2$ is a special hyperplane in parameter space [54] where the dimensionality of the phase space is reduced to two [55]. This requires a separate analysis and so we will first treat the general three-dimensional phase space dynamics and return to this later.

Next, one can express some useful quantities in terms of these variables:

$$
\begin{aligned}
\Omega_{\phi} & =\frac{\dot{\phi}_{\infty}^{2}}{6 H^{2}}+\frac{V(\phi)}{3 H^{2}}=x^{2}+y^{2} \\
\omega_{\phi} & =\frac{\dot{\phi}_{\infty}^{2}-2 V}{\dot{\phi}_{\infty}^{2}+2 V}=\frac{x^{2}-y^{2}}{x^{2}+y^{2}} \\
D & \equiv \frac{2 B^{2} X}{\Lambda^{2}}=6 x^{2} z^{2} \text { and } \\
Q & =\frac{\alpha+z^{2}\left[\sqrt{6} x+6 x^{2}(\gamma-\alpha)+3 \sqrt{\frac{3}{2}} x\left(y^{2}-x^{2}-1\right)\right]}{1-6 x^{2} z^{2}} .
\end{aligned}
$$

$\Omega_{\phi} \sim 1$ corresponds to a dark energy dominated solution, $Q=0$ corresponds to the absence of any fifth forces on small scales and $D=1$ signifies a singularity in the Jordan frame metric [which is proportional to $(1-D)^{1 / 2}$ ].
The problem of finding dark energy dominated accelerating solutions where fifth forces are absent is then reduced to finding stable fixed points of the autonomous system where $\Omega_{\phi}$ and $\omega_{\phi}$ are compatible with current dark energy observations and $Q=0$. We treat solutions where $D=1$ as unviable for the reasons explained in Sec. II A.

\section{B. Fixed points}

Before studying the new system (32)-(34), it is worth recalling some useful properties of the equivalent system when only a conformal coupling is present, which corresponds to $z=0$. This was studied by [35] (see also [36]). There are two stable fixed points:

(i) Dark energy dominated fixed point: This has $\Omega_{\phi}=1$ and $\omega_{\phi}=-1+\lambda^{2} / 3$. It exists whenever $\lambda<\sqrt{6}$ and is stable whenever $\lambda<\left(\sqrt{\alpha^{2}+12}-\alpha\right) / 2$.

(ii) Variable fixed point: This may or may not give dark energy domination depending on the choice of parameters, although solutions which match the current observations suffer from the lack of a matter-dominated era, at least when $\alpha$ and $\lambda$ are constant [56]. It is always stable but only exists when the dark energy dominated solution is unstable and is hence the only stable fixed point when $\lambda>\sqrt{6}$. There is a critical value of $\alpha$ below which it is a stable node and above which it is a stable spiral.

In the case of a purely conformal coupling, the phase space is a semicircle in the $x-y$ plane [we do not consider $V(\phi)<0]$. Since $z>0(B>0$ for stability reasons, see [17]) the phase space of the disformal system is an infinite semicircular prism restricted to the upper half of the $z$ plane $(z>0)$. This parametrization could be problematic because $z$ can potentially reach $\infty$ and so there may be fixed points at infinity. Indeed, this is the case but we will analyze the fixed points of the system (32)-(34) first in order to make contact with the purely conformal case. Setting Eqs. (32)-(34) equal to zero, we find the fixed points given in Table I. The cosmological relevant parameters are given in Table II. Points (1)-(5) are the fixed points found by [35] for the purely conformal case; they have $z=0$ so that all disformal effects are absent [57]. Note also that these points have $Q=\alpha$ and hence lead to large unscreened fifth forces on all scales unless one tunes this to very small values. Since $D=0$ at these points the Jordan frame metric is nonsingular at late times.

The new points are (6) and (7), which are both unviable as cosmological solutions. These points behave as a stiff fluid $\left(\omega_{\phi}=1\right)$ and so cannot describe a dark energy dominated universe. Furthermore, point (7) is unphysical except for the special point $\gamma=\sqrt{3 / 2}$; when $\gamma>\sqrt{3 / 2}$ one has $\Omega_{\phi}>1$ and the point lies outside the physical state space. Even if this were not the case, they have $D=1$ signaling a metric singularity in the infinite future and are unviable for the reasons discussed in Sec. II A. 
TABLE I. The fixed points of the system (32)-(34) with $\gamma \neq \lambda / 2$.

\begin{tabular}{|c|c|c|c|c|}
\hline Name & $x$ & $y$ & $z$ & Existence \\
\hline (1) & $-\sqrt{\frac{2}{3}} \alpha$ & 0 & 0 & $\alpha<\sqrt{3 / 2}$ \\
\hline (2) & -1 & 0 & 0 & All \\
\hline (3) & 1 & 0 & 0 & All \\
\hline (4) & $\frac{\lambda}{\sqrt{6}}$ & $\sqrt{1-\frac{\lambda^{2}}{6}}$ & 0 & $\lambda<\sqrt{6}$ \\
\hline (5) & 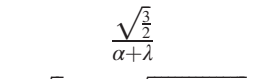 & $\frac{\sqrt{3+2 \alpha(\alpha+\lambda)}}{\sqrt{2}(\alpha+\lambda)}$ & 0 & $\alpha>3 / \lambda-\lambda$ \\
\hline (6) & $\sqrt{\frac{2}{3}} \gamma-\sqrt{\frac{2}{3} \gamma^{2}-1}$ & 0 & $\frac{1}{3} \sqrt{2 \gamma^{2}+\gamma \sqrt{4 \gamma^{2}-6}-\frac{3}{2}}$ & $\gamma>\sqrt{3 / 2}$ \\
\hline (7) & $\sqrt{\frac{2}{3}} \gamma+\sqrt{\frac{2}{3} \gamma^{2}-1}$ & 0 & $\frac{1}{3} \sqrt{2 \gamma^{2}-\gamma \sqrt{4 \gamma^{2}-6}-\frac{3}{2}}$ & $\gamma=\sqrt{3 / 2}$ \\
\hline
\end{tabular}

TABLE II. The cosmological quantities at the fixed points of the system (32)-(34) with $\gamma \neq \lambda / 2$. Models with $D=1$ do not have a corresponding value of $Q$ since it is not determined uniquely by the properties of the fixed point (see the discussion in Sec. II A).

\begin{tabular}{lcccc}
\hline \hline Name & $Q$ & $\Omega_{\phi}$ & $\omega_{\phi}$ & $D$ \\
\hline (1) & $\alpha$ & 1 & 1 & 0 \\
$(2)$ & $\alpha$ & 1 & 1 & 0 \\
$(3)$ & $\alpha$ & $\frac{2 \alpha^{2}}{3}$ & 1 & 0 \\
(4) & $\alpha$ & 1 & $-1+\frac{\lambda^{2}}{3}$ & 0 \\
$(5)$ & $\alpha$ & $\frac{3+\alpha(\alpha+\lambda)}{(\alpha+\lambda)^{2}}$ & $1+\frac{3}{3+\alpha(\alpha+\lambda)}$ & 0 \\
$(6)$ & - & $\frac{1}{3}\left(\sqrt{-3+2 \gamma^{2}}-\sqrt{2} \gamma\right)^{2}$ & 1 & 1 \\
(7) & - & $\frac{1}{3}\left(\sqrt{-3+2 \gamma^{2}}+\sqrt{2} \gamma\right)^{2}$ & 1 & 1 \\
\hline \hline
\end{tabular}

Since the dimension of the phase space is increased from the purely conformal case, the stability of the fixed points is altered; there are now three eigenvalues instead of two. In particular, whereas the purely conformal fixed points [(1)-(5)] do not depend on $\gamma$, their stability does.

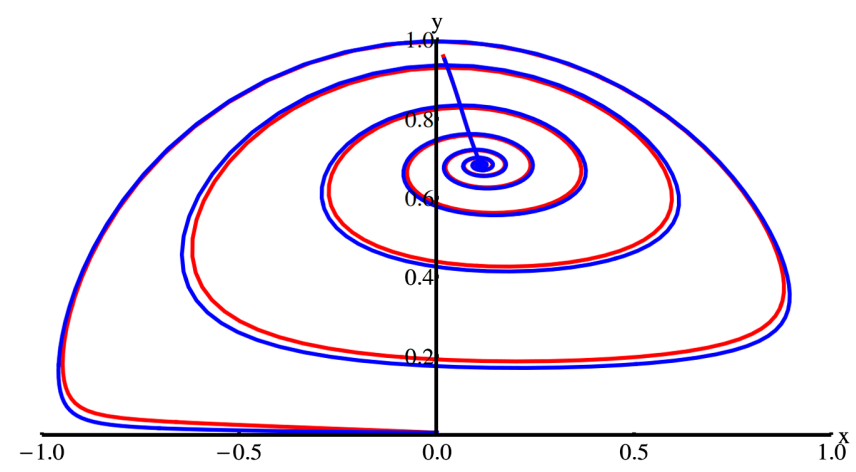

FIG. 1 (color online). The trajectories in the $x-y$ plane for $\alpha=5, \gamma=4$ and $\lambda=6$ with initial conditions $\phi_{0}=-1$ and $\phi_{0}^{\prime}=0$. The red track corresponds to $H_{0} / \Lambda=10^{-2}$ and the blue track to $H_{0} / \Lambda=10^{-3}$.
The eigenvalues $e_{1,2,3}$ for each fixed point are given in Appendix B. One may check that there are choices of the parameters $\alpha, \gamma$ and $\lambda$ where none of these fixed points are stable. Indeed, one may check that when $\alpha=5, \gamma=4$ and $\lambda=6$, all of the fixed points are either unstable or saddle points. The trajectory in the $x-y$ plane of solutions with $H_{0} / \Lambda=10^{-2}$ and $10^{-3}$ and initial conditions $\phi_{0}=-1$ and $\phi_{0}^{\prime}=0$ is shown in Fig. 1. Furthermore, we show the evolution of $(1-D)$ in Fig. 2. One can see that in each case, the system spends a long time near the saddle point before moving towards $x=0, y=1$. Along this trajectory, $(1-D)$ approaches zero so that the Universe is tending to a state where the Jordan frame metric is singular. Furthermore, one can see that this behavior is independent of the choice of $\Lambda$ and one can check that it is not affected by the initial conditions either. This suggests that the system has a common late-time behavior not captured by the fixed-point analysis above. The next subsection is devoted to understanding this.

\section{Fixed points at infinity}

Numerically, one finds that a continued integration of the system to later times pushes $x$ closer to $0, y$ closer to 1 and $z$

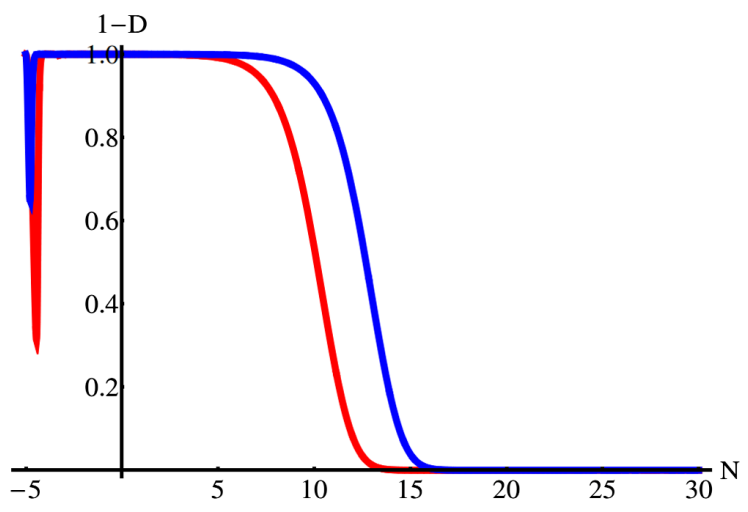

FIG. 2 (color online). $\quad 1-D$ as a function of $N$ for the models described in Fig. 1. Recall that $\sqrt{-\tilde{g}}=(1-D)^{1 / 2} \sqrt{-g}$ and so $D=1$ represents a metric singularity of the Jordan frame metric. 
to increasingly larger values. This suggests that the behavior may be due to fixed points at $z=\infty$ and so we investigate these by compactifying the phase space. Defining

$$
Z \equiv \frac{z}{z+1}
$$

so that $0 \leq Z \leq 1$ we can map the points at $z=\infty$ to $Z=1$ while $z=0$ corresponds to $Z=0$. The phase space in these coordinates is then the finite semicircular prism. In these coordinates, the system is described by the three first-order equations

$$
\frac{\mathrm{d} x}{\mathrm{~d} N}=-3 x+\sqrt{\frac{3}{2}} \lambda y^{2}+\frac{3}{2} x\left(x^{2}-y^{2}+1\right)+\sqrt{\frac{3}{2}} \frac{\left(x^{2}+y^{2}-1\right)\left(6 x^{2} Z^{2}(\alpha-\gamma)+3 \sqrt{6} x Z^{2}-3 \lambda y^{2} Z^{2}-\alpha(Z-1)^{2}\right)}{Z^{2}\left(9 x^{2}+3 y^{2}-4\right)+2 Z-1},
$$

$$
\begin{aligned}
& \frac{\mathrm{d} y}{\mathrm{~d} N}=\frac{3}{2} y\left(1+x^{2}-y^{2}\right)-\sqrt{\frac{3}{2}} x y \lambda \text { and } \\
& \frac{\mathrm{d} Z}{\mathrm{~d} N}=\frac{1}{2}(Z-1) Z\left(3 x^{2}-2 \sqrt{6} \gamma x-3 y^{2}+3\right) .
\end{aligned}
$$

In addition to the fixed points found above, one finds the fixed points given in Table III [58]. The cosmological parameters at each point are shown in Table IV. We have omitted point (11) since this is discussed in detail in the next section. The eigenvalues are listed in Appendix A 2 but here we note that the eigenvalues for point (11) are

$$
e_{1}=-3, \quad e_{2}=0 \quad \text { and } \quad e_{3}=0
$$

\section{Center manifold analysis}

The two zero eigenvalues indicate that a linear analysis is not sufficient to determine the stability of the system near this fixed point. In order to do this, one must perform a center manifold analysis. This has been used in cosmological systems previously (see [59] for a recent application to $m^{2} \phi^{2}$ potentials and references therein for further examples) and we give a full account for the unfamiliar reader in Appendix A 2. For simplicity, we move the point to the origin by making the change of variables:

$$
\begin{aligned}
& Z=W+1 \text { and } \\
& y=Y+1 .
\end{aligned}
$$

We will not give the new first-order system here but for completeness it is given in Appendix C. This change of variables does not change the eigenvalues but does change the eigenvectors, which, in this basis, are

$$
\vec{e}_{1}=\left(\begin{array}{l}
0 \\
1 \\
0
\end{array}\right), \quad \vec{e}_{2}=\left(\begin{array}{l}
0 \\
0 \\
1
\end{array}\right) \quad \text { and } \quad \vec{e}_{3}=\left(\begin{array}{c}
-\frac{\sqrt{6}}{\lambda} \\
1 \\
0
\end{array}\right) \text {, }
$$

TABLE III. The fixed points of the compactified system (42)-(44). Fixed points with $Z=0$ are not shown since they are already present in Table I. Similarly, fixed points (4) and (5) are not shown. We have listed the condition for the existence of the fixed point but that is not to say that it lies inside the physical phase space; one should check with Table IV to ensure that the cosmological parameters, especially $\Omega_{\phi}$, assume values inside the physical state space.

\begin{tabular}{lcccc}
\hline \hline Name & $x$ & $y$ & $Z$ & Existence \\
\hline$(8)$ & -1 & 0 & 1 & All \\
$(9)$ & 0 & 0 & 1 & All \\
$(10)$ & 1 & 0 & 1 & All \\
$(11)$ & 0 & 1 & 1 & All \\
$(12)$ & $-\frac{\sqrt{2(\alpha-\gamma)^{2}-9}+\sqrt{2} \alpha-\sqrt{2} \gamma}{3 \sqrt{3}}$ & 0 & 1 & $\alpha-\gamma>\frac{3}{\sqrt{2}}$ \\
$(13)$ & $\frac{\sqrt{2(\alpha-\gamma)^{2}-9}-\sqrt{2} \alpha+\sqrt{2} \gamma}{3 \sqrt{3}}$ & 0 & 1 & $\alpha-\gamma>\frac{3}{\sqrt{2}}$ \\
$(14)$ & $\frac{\lambda}{\sqrt{6}}$ & $\sqrt{1-\frac{\lambda^{2}}{6}}$ & 1 & $\lambda<\sqrt{6}$ \\
$(15)$ & $\frac{\sqrt{6}}{2 \alpha-2 \gamma+3 \lambda}$ & $\sqrt{\frac{6}{2 \alpha-2 \gamma+2 \alpha-2 \gamma+3}}$ & 1 & $2 \alpha-2 \gamma+3 \lambda>0$ \\
$(16)$ & $\frac{\sqrt{4 \gamma^{2}-6}+2 \gamma}{\sqrt{6}}$ & 0 & $\frac{1}{\sqrt{4 \gamma^{2}-6}+2 \gamma+1}$ & $\gamma>\sqrt{\frac{2}{3}}$ \\
$(17)$ & $\frac{2 \gamma-\sqrt{4 \gamma^{2}-6}}{\sqrt{6}}$ & 0 & $\frac{\sqrt{4 \gamma^{2}-6+2 \gamma+1}}{4 \gamma+7}$ & $\gamma>\sqrt{\frac{2}{3}}$ \\
\hline \hline
\end{tabular}


TABLE IV. The cosmological quantities at the fixed points of the compactified system. Note that $Q \rightarrow \pm \infty$ reflects the fact that $z \rightarrow \infty$. The sign depends on the choice of $\gamma, \lambda$ and $\alpha$. Models with $D=1$ do not have a corresponding value of $Q$ since it is not determined uniquely by the properties of the fixed point (see the discussion in Sec. II A).

\begin{tabular}{|c|c|c|c|c|}
\hline Name & $Q$ & $\Omega_{\phi}$ & $\omega_{\phi}$ & $D$ \\
\hline (8) & $\pm \infty$ & 1 & 1 & $\infty$ \\
\hline (9) & $\alpha$ & 0 & 0 & $\infty$ \\
\hline (10) & $\pm \infty$ & 1 & 1 & $\infty$ \\
\hline (12) & $\pm \infty$ & $\frac{1}{27}\left(\sqrt{2(\alpha-\gamma)^{2}-9}+\sqrt{2} \alpha-\sqrt{2} \gamma\right)^{2}$ & 1 & $\infty$ \\
\hline (13) & $\pm \infty$ & $\frac{1}{27}\left(\sqrt{2(\alpha-\gamma)^{2}-9}+\sqrt{2} \alpha-\sqrt{2} \gamma\right)^{2}$ & 1 & $\infty$ \\
\hline (14) & $\pm \infty$ & 1 & $-1+\lambda^{2} / 3$ & $\infty$ \\
\hline$(15)$ & $\pm \infty$ & $\frac{4 \alpha^{2}-8 \alpha \gamma+8 \alpha \lambda+4 \gamma^{2}-8 \gamma \lambda+3 \lambda^{2}+12}{(2 \alpha-2 \gamma+3 \lambda)^{2}}$ & $-\frac{(2 \alpha-2 \gamma+\lambda)(2 \alpha-2 \gamma+3 \lambda)}{4 \alpha^{2}-8 \alpha \gamma+8 \alpha \lambda+4 \gamma^{2}-8 \gamma \lambda+3 \lambda^{2}+12}$ & $\infty$ \\
\hline (16) & - & $\frac{1}{6}\left(\sqrt{4 \gamma^{2}-6}+2 \gamma\right)^{2}$ & 1 & 1 \\
\hline$(17)$ & - & $\frac{1}{6}\left(\sqrt{4 \gamma^{2}-6}-2 \gamma\right)^{2}$ & 1 & 1 \\
\hline
\end{tabular}

where the first is an eigenvalue -3 vector and the final two are zero eigenvectors. As discussed in Appendix A, perturbations about the fixed point will evolve according to

$$
\left(\begin{array}{c}
\delta x \\
\delta Y \\
\delta W
\end{array}\right)=\vec{B}_{1} e^{e_{1} t}+\vec{B}_{2} e^{e_{2} t}+\vec{B}_{3} e^{e_{3} t}
$$

where $\vec{B}_{i}$ are the vectors that span the eigenbasis of the matrix $M_{i j}$ defined in (A3), which is found by expanding the equations to linear order in small perturbations $\{\delta x, \delta Y, \delta W\}$. One can see that zero eigenvalues neither grow nor decay at linear order; one must go to the next-toleading order to determine the dynamics. This means that for any initial configuration sufficiently close to the fixed point, the trajectory along the $\vec{e}_{1}$ direction will rapidly tend to its fixed-point value whereas the trajectory in the plane described by $\vec{e}_{1,2}$ - the center manifold-will evolve on a slower time scale. The essence of the center manifold technique is to find the new variable along the $\vec{e}_{1}$ direction and assume that its evolution equation is already minimized. One can then solve this to obtain an algebraic expression for this variable in terms of those parametrizing the center manifold. The equations for the other two variables-the center variables - then describe the dynamics in the center manifold at late times. One has then reduced the dimension of the phase space to that of the center manifold. Writing

$$
\left(\begin{array}{c}
x \\
Y \\
W
\end{array}\right)=\sum_{i} A_{i} \vec{e}_{i}
$$

one finds

$$
\left(\begin{array}{c}
x \\
Y \\
W
\end{array}\right)=\left(\begin{array}{c}
-\frac{\sqrt{6}}{\lambda} A_{3} \\
A_{1}+A_{3} \\
A_{2}
\end{array}\right)
$$

which can be inverted to give

$$
\left(\begin{array}{c}
A_{1} \\
A_{2} \\
A_{3}
\end{array}\right)=\left(\begin{array}{c}
Y+\frac{\lambda}{\sqrt{6}} x \\
W \\
-\frac{\lambda}{\sqrt{6}} x
\end{array}\right)
$$

Setting $A_{1}^{\prime}=0$ i.e. the variable along $\vec{e}_{1}$, one finds

$$
\frac{\mathrm{d} y}{\mathrm{~d} x}=-\frac{\lambda}{\sqrt{6}} .
$$

Therefore, at late times, when the system evolves towards fixed point (11) the trajectory in the $x-y$ plane is

$$
y \approx 1-\frac{\lambda}{\sqrt{6}} x .
$$

In Fig. 3 we plot the same models as shown in Fig. 1 and overlay this line. One can see that the trajectories indeed converge to this at late times once they have left the saddle point.

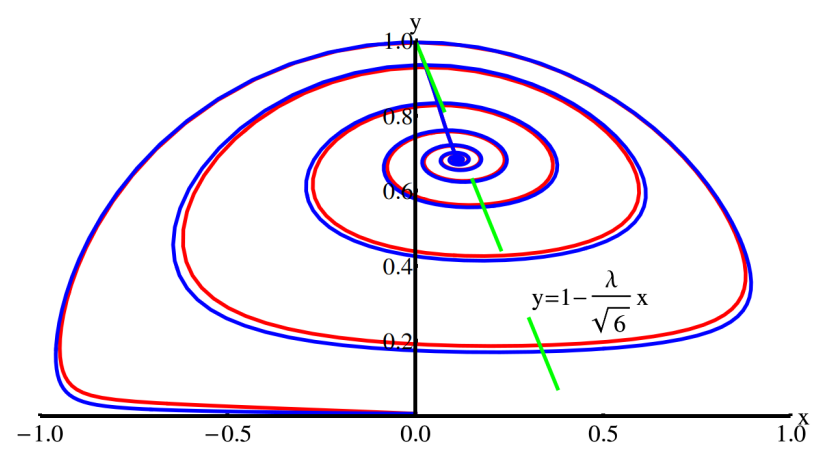

FIG. 3 (color online). The $x-y$ plane for the same models as Fig. 1 but overlaid with the center manifold prediction for the late-time trajectories $y=1-\lambda x / \sqrt{6}$ (green dashed line). 
Finally, one can examine the behavior of the determinant of the Jordan frame metric along this trajectory. We begin with the equations for the center variables, where we have set $A_{1}=0$, which follows from the values of $x$ and $Y$ at the fixed point:

$$
\begin{aligned}
\frac{\mathrm{d} A_{2}}{\mathrm{~d} N}= & -\frac{3 A_{2}\left(A_{2}+1\right) A_{3}\left(A_{3}\left(\lambda^{2}-6\right)+2 \lambda(\lambda-2 \gamma)\right)}{2 \lambda^{2}} \text { and } \\
\frac{\mathrm{d} A_{3}}{\mathrm{~d} N}= & -\frac{\lambda A_{3}\left(\frac{6 A_{3}}{\lambda^{2}}+A_{3}+2\right)\left(\left(A_{2}+1\right)\left(2 \alpha+\left(A_{2}+1\right)\left(-\alpha+\frac{18 A_{3}\left(2 A_{3}(\alpha-\gamma)-\lambda\right)}{\lambda^{2}}-3\left(A_{3}+1\right)^{2} \lambda\right)\right)-\alpha\right)}{2\left(A_{2}+1\right)\left(\left(A_{2}+1\right)\left(A_{3}^{2}\left(\frac{54}{\lambda^{2}}+3\right)+6 A_{3}-1\right)+2\right)-2} \\
& -\frac{3 A_{3}^{2}\left(A_{3}\left(\lambda^{2}-6\right)+2 \lambda^{2}\right)}{2 \lambda^{2}}-3 A_{3}-\frac{\left(A_{3}+1\right)^{2} \lambda^{2}}{2} .
\end{aligned}
$$

One can then find the fixed points of this reduced phase space. The only two physical fixed points are

$$
A_{2}=\frac{12(\lambda-2 \gamma)}{24 \gamma \pm \lambda^{2}-12 \lambda \pm 6} \text { and } A_{3}=\frac{2 \lambda(2 \gamma-\lambda)}{\lambda^{2}-6}
$$

In these coordinates, one has

$$
D=1-\frac{36 A_{3}^{2}\left(A_{2}+1\right)^{2}}{\lambda^{2} A_{2}^{2}}
$$

and one can verify that this is indeed zero at these fixed points. Therefore, any trajectory in the $x-y$ plane that evolves towards this fixed point will evolve in such a way that $\sqrt{-\tilde{g}} \rightarrow 0$ but only in the infinite future. This is the underlying reason for the pathology resistance discussed above [60]: the system will evolve towards a metric singularity but only in the infinite future and therefore any trajectory must necessarily slow down as it is approached. To date, this feature has been observed numerically but no underlying reason has been discerned. What we have shown here is that this is a generic feature of models where the conformal fixed points are all unstable. As discussed in Sec. II A, models that exhibit this singularity are not viable since they suffer from large fifth forces and may not have a viable nonrelativistic limit and so we conclude that in the general case, the only viable models are the disformal generalizations of the two conformal fixed points [(4) and (5)]. Therefore, one requires either $\alpha \ll 1$ or that the visible sector is coupled to the Einstein frame metric directly in order to avoid fifth forces. Furthermore, the late-time properties of these models are identical to those of the equivalent purely conformal theory, although the dynamics before the fixed point are reached may be different.

\section{REDUCED PHASE SPACE}

In this section, we examine the special parameter choice $\gamma=\lambda / 2$, which we argued above reduces the dimension of the phase space to two and therefore requires a separate analysis. We can choose to eliminate either $z$ or $y$ from the equations and we choose to eliminate $z$ in order to make contact with the purely conformal case. Furthermore, $z \rightarrow \infty$ corresponds to $y \rightarrow 0$ and so this substitution captures all of the new fixed points without having to compactify further. Using Eq. (36) in Eqs. (32) and (33) we find the following two-dimensional autonomous system for $x$ and $y$ :

$$
\begin{aligned}
\frac{\mathrm{d} x}{\mathrm{~d} N}= & \sqrt{\frac{3}{2}} \frac{\left(x^{2}+y^{2}-1\right)\left(x^{2}(2 \alpha-\lambda)+\sqrt{6} x-y^{2}\left(\alpha \tilde{\Lambda}^{2}+\lambda\right)\right)}{3 x^{2}-\left(\tilde{\Lambda}^{2}-1\right) y^{2}-1} \\
& +\frac{3}{2} x\left(x^{2}-y^{2}+1\right)-3 x+\sqrt{\frac{3}{2}} \lambda y^{2},
\end{aligned}
$$

where the second equation is (33) and $\tilde{\Lambda} \equiv \Lambda / m_{0}$. The fixed points which lie inside the physical phase space are shown in Table $\mathrm{V}$ and the cosmological quantities at these points are shown in Table VI. Note that one has a choice of

TABLE V. The fixed points of the system when $\gamma=\lambda / 2$. Note that the existence indicates where the point exists; one may find parameter choices where the point exists but lies outside the physical state space. Note that point (20) exists for all $\tilde{\Lambda}$ when $\lambda>\sqrt{3}$. When the converse is true one can find a narrow region in the $\tilde{\Lambda}-\lambda$ plane where the point still exists. We will not be interested in this region in this work.

\begin{tabular}{lccc}
\hline \hline Name & $x$ & $y$ & Existence \\
\hline$(18)$ & $\frac{\lambda}{\sqrt{6}}$ & $\sqrt{1-\frac{\lambda^{2}}{6}}$ & $\lambda<\sqrt{6}$ \\
$(19)$ & $\frac{\sqrt{3}}{\alpha+\lambda}$ & $\frac{\sqrt{3+2 \alpha(\alpha+\lambda)}}{\sqrt{2}(\alpha+\lambda)}$ & $\alpha>3 / \lambda-\lambda$ \\
$(20)$ & $\frac{\lambda \tilde{\Lambda}^{2}-\sqrt{\left(\lambda^{2}-6\right) \tilde{\Lambda}^{4}+12 \tilde{\Lambda}^{2}}}{\sqrt{6}\left(\tilde{\Lambda}^{2}-2\right)}$ & $\sqrt{6}\left(\left(\lambda^{2}-3\right) \tilde{\Lambda}^{2}+\lambda \sqrt{\tilde{\Lambda}^{2}\left(\left(\lambda^{2}-6\right) \tilde{\Lambda}^{2}+12\right)}+6\right)$ & $\lambda>\sqrt{3}$ \\
\hline \hline
\end{tabular}


TABLE VI. The cosmological quantities at the fixed points of the system when $\gamma=\lambda / 2$. Models with $D=1$ do not have a corresponding value of $Q$ since it is not determined uniquely by the properties of the fixed point (see the discussion in Sec. II A).

\begin{tabular}{|c|c|c|c|c|}
\hline Name & $Q$ & $\Omega_{\phi}$ & $\omega_{\phi}$ & $D$ \\
\hline (18) & $\frac{\alpha\left(\lambda^{2}\left(\tilde{\Lambda}^{2}+2\right)-6 \tilde{\Lambda}^{2}\right)}{\left(\lambda^{2}-6\right) \tilde{\Lambda}^{2}}$ & 1 & $-1+\frac{\lambda^{2}}{3}$ & $-\frac{2 \lambda^{2}}{\left(\lambda^{2}-6\right) \tilde{\Lambda}^{2}}$ \\
\hline (19) & $\alpha-\frac{6}{\tilde{\Lambda}^{2}\left(2 \alpha^{2}+2 \alpha \lambda+3\right)}$ & $\frac{\alpha(\alpha+\lambda)+3}{(\alpha+\lambda)^{2}}$ & $-1+\frac{3}{3+\alpha(\alpha+\lambda)}$ & $\frac{6}{\tilde{\Lambda}^{2}(2 \alpha(\alpha+\lambda)+3)}$ \\
\hline (20) & - & $\frac{3\left(\tilde{\Lambda}^{2}+2\right)}{\left(\lambda^{2}-3\right) \tilde{\Lambda}^{2}+\lambda \sqrt{\left(\lambda^{2}-6\right) \tilde{\Lambda}^{4}+12 \tilde{\Lambda}^{2}}+6}$ & $1-\frac{4}{\tilde{\Lambda}^{2}+2}$ & 1 \\
\hline
\end{tabular}

minimizing either the $x-y$ or $x-z$ system. Either is fine, but it is important to note that the redundant equation acts as a constraint and should be identically zero at the fixed points. When this is not the case the points are unphysical and we do not include them. Points (18) and (19) are points (4) and (5), although they have different scalar charges and their stability is different; the interesting point is (20). We list the eigenvalues in Appendix B. The stability of point (18) is identical to the conformal case but the stability of (19) is altered from both the conformal case and the case where $\gamma \neq \lambda / 2$. Furthermore, one can check that point (20) is stable over a large range of parameter space and is often simultaneously stable when point (18) is. When point (18) does not exist, it is often the case that point (19) is unstable and (20) is the only stable attractor of the system.

Interestingly, this fixed point can match the current observations of dark energy. To see this, consider as an example the WMAP9 results [37], $\omega=-0.97, \Omega_{\mathrm{DE}}=$ 0.704 [61]. One finds that fixed point $(20)$ can reproduce this exactly by taking $\lambda=3.77953$ and $\tilde{\Lambda}=0.174519$ with $\alpha$ arbitrary. As an example, consider the case $\alpha=2$. In the purely conformal case the system would evolve to the variable fixed point where $\Omega_{\phi} \approx 0.436$ and $\omega_{\phi} \approx-0.794$, which are far from the WMAP values. In Fig. 4 we plot the $x-y$ plane for both the purely conformal case and the disformal case with the same initial conditions. One can see

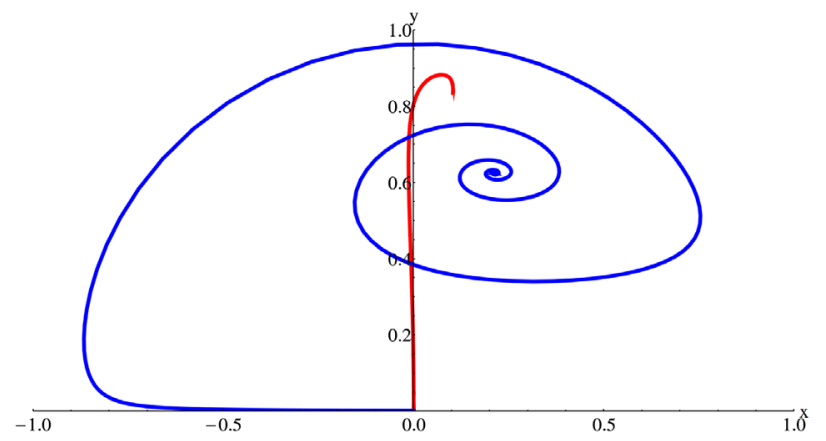

FIG. 4 (color online). The trajectories in the $x-y$ plane for a purely conformal theory with $\alpha=2$ (blue) and a disformal theory with $\gamma=\lambda / 2$ (red). In each case the initial conditions are $\phi_{0}=$ -1 and $\phi_{0}^{\prime}=0$. The parameters $\Lambda, m_{0}$ and $\lambda$ were chosen such that the disformal attractor corresponds to a universe where $\omega_{\phi}$ and $\Omega_{\phi}$ match the WMAP9 observations. that the disformal trajectory converges to fixed point (20) whereas the purely conformal case reaches fixed point (18) (or rather, the conformal equivalent). To illustrate this further, we plot $\Omega_{\phi}$ and $\omega_{\phi}$ in Figs. 5 and 6. It is interesting to note that, unlike the purely conformal case where there is a lack of a matter-dominated era [56], the disformal system delays the onset of the field rolling and allows for a period

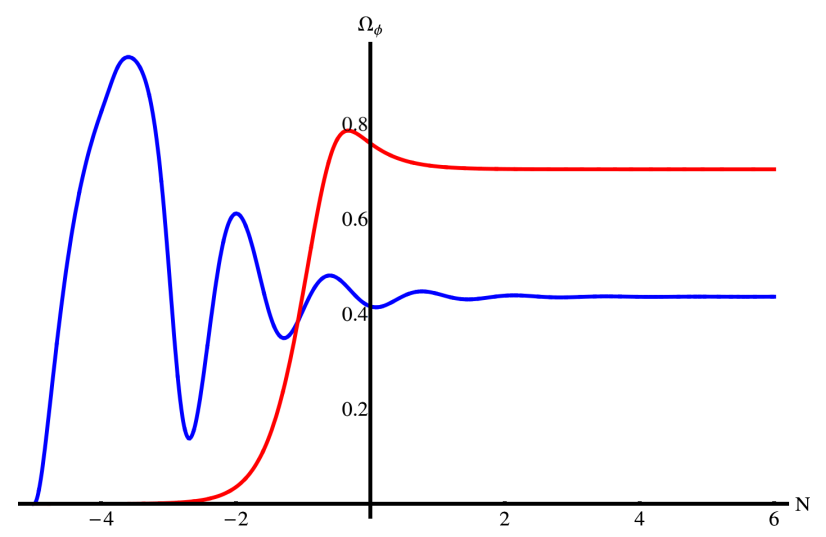

FIG. 5 (color online). $\Omega_{\phi}$ as a function of $N$ for a purely conformal theory with $\alpha=2$ (blue) and a disformal theory with $\gamma=\lambda / 2$ (red). The parameters and initial conditions are as indicated in Fig. 4's caption.

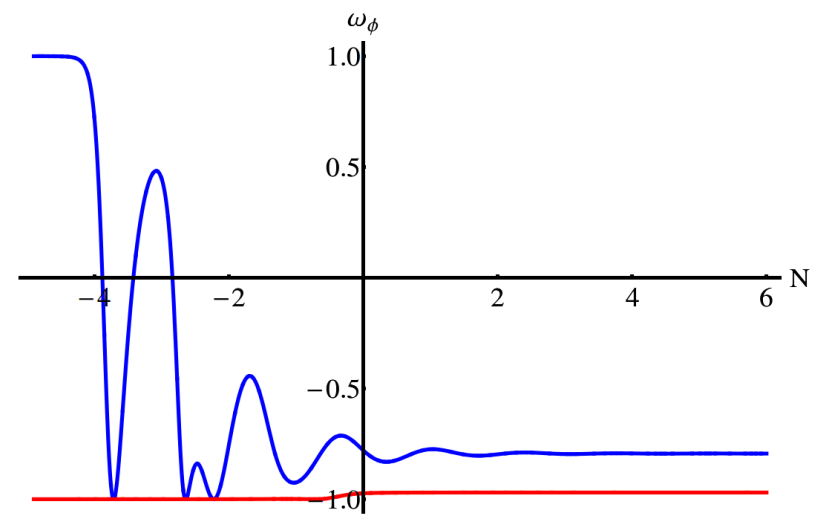

FIG. 6 (color online). $\omega_{\phi}$ as a function of $N$ for a purely conformal theory with $\alpha=2$ (blue) and a disformal theory with $\gamma=\lambda / 2$ (red). The parameters and initial conditions are as indicated in Fig. 4's caption. 
of matter domination. This behavior was also noted in [21]. Finally, one can see that the fixed point has $D=1$, and so there is a singularity of $\sqrt{-\tilde{g}}$ in the infinite future. This fixed point is then unviable and we are left with points (18) and (19), the disformal equivalent of points (4) and (5). Even though the scalar charge differs from $\alpha$, one still requires $\alpha \ll 1$ for these points to be viable. If one tunes the model parameters such that $\tilde{\Lambda}^{2}=2 \lambda^{2} /\left(6-\lambda^{2}\right)$ so that the scalar charge at point (18) is zero then one finds $D=1$ so that the metric singularity is present and the model is unviable. One is then left with tuning $\alpha \ll 1$ or decoupling the scalar from visible matter. Tuning the parameters so that the scalar charge at point (19) vanishes sets $D=\alpha$ and we must again tune $\alpha \ll 1$ in order to avoid the pathologies associated with the metric singularity.

\section{DISCUSSION AND CONCLUSIONS}

In this work we have studied the solution space of disformal theories of gravity using dynamical systems techniques. Our ultimate goal was to find stable dark energy dominated solutions where the local scalar charge is zero at late times. The Jordan frame metric can become singular, indicating a potential instability of the theory. Several previous works have noted a natural resistance to pathology where numerical solutions show that the field slows down as the singularity is approached. Here, we were able to explain this by showing that any singularity is only reached in an infinite amount of time. Despite this, we argued that models that exhibit this behavior are still unviable (at least when the scalar couples to the visible sector) due to large unscreened fifth forces and that such a singularity may indicate the lack of an appropriate nonrelativistic limit.

We showed that, in the general case, the phase space of the system is three dimensional rather than the twodimensional phase space of the equivalent quintessence and purely conformal theories. It was found that there are no new viable fixed points of the system and furthermore there are parameter choices where the attractors that are reached in the purely conformal case are now saddle points. This is due to the increased dimension of the phase space, which changes the characteristic polynomial for the eigenvalues to a cubic equation rather than a quadratic, yielding a third solution that can be positive. Numerically, we observed that when the parameters assume these values, the trajectories spend a long time near the saddle points before evolving towards a dark energy dominated fixed point. The Jordan frame becomes singular along the approach to this fixed point and hence these models are not viable. Two of the eigenvalues at this fixed point are zero and so it was necessary to use center manifold techniques to discern the late-time behavior.

We identified a tuning in parameter space, $\gamma=\lambda / 2$, where the dimension of the phase space is reduced to two and therefore the dynamics are altered. There is a fixed point — point (20) — of the reduced phase space not present in the purely conformal system. This point is a stable attractor and can match the presently observed dark energy parameters by tuning $\lambda$ and $\tilde{\Lambda}=\Lambda / m_{0}$. In particular, by taking $\lambda=3.77953$ and $\tilde{\Lambda}=0.174519$ we were able to reproduce the WMAP9 density parameter and equation of state. Unfortunately, this fixed point is not viable since the Jordan frame metric becomes singular in the infinite future.

We therefore conclude that the only viable models of disformal dark energy have late-time properties to the equivalent models where the disformal coupling is absent. Furthermore, since these models are not stable over the entire parameter space, the inclusion of a disformal factor greatly reduces the choice of allowed model parameters. When these models are not stable, the cosmological solutions evolve towards fixed points where the Jordan frame metric is singular and include all the pathologies associated with this. The inclusion of a disformal factor seems to have little to say about dark energy and its inclusion introduces further problems that require reducing the viable parameter space to solve. With this in mind, it is worth raising the question of whether it is worthwhile to pursue these theories as dark energy candidates further.

In this work we have only considered theories where all matter couples to the Jordan frame metric universally but this does not necessarily have to be the case and, as we have remarked above several times already, one could alleviate some of the problems associated with the metric singularity by coupling visible matter to the Einstein frame metric. In this case, the issue of fifth forces is moot since objects in the Solar System do not feel the fifth force and there is no ambiguity as to how the coordinate time is related to the clock used by static observers. Furthermore, since the evolution of the Universe in the matter era is driven by dark matter and not baryons, some of the fixed points considered unviable here may be viable in these more general theories. Of particular interest is fixed point (20), which can match the presently observed dark energy density and equation of state by tuning only two of the four free parameters (three if one counts the $\gamma=\lambda / 2$ tuning). Whether or not this scenario is viable requires further investigation beyond the scope of this work. Dark matter particles will see an effective value of Newton's constant given by $\left(1+2 Q^{2}\right) G$, which becomes infinite as the fixed point is approached and so one expects to see novel effects in the cold dark matter power spectrum and collapsed halos. Another potential issue is whether the apparent lack of a nonrelativistic limit is incompatible with the notion of nonrelativistic cold dark matter altogether.

Here we have taken the simplest model where $\gamma, \lambda$ and $\alpha$ are constants. The dynamics are altered when one allows them to vary. One can then investigate the dynamics of the new system, which may have a dimension larger than three, or try to find models where the late-time fixed points give 
the observed dark energy parameters. In terms of our model, the choice $\gamma=\lambda / 2$ was a parameter tuning but for more general models one requires that $\gamma(\phi)=\lambda(\phi) / 2$ is reached dynamically. It remains to be seen if one can contrive more general models where this is achieved. One may also worry that quantum corrections can spoil this tuning; this certainly merits investigation.

Only recently have the properties of disformal theories been fully elucidated and, unlike more well-studied models, there is no canonical disformal paradigm. Indeed, to date, a viable model that screens fifth forces and can account for all cosmological observations is still lacking. What we have done here is to classify the possible cosmological solutions and identify a class of models - albeit using a special tuning - that can reproduce the observed dark energy parameters today. It is unlikely that this solution is viable when the scalar couples to visible matter and so the study of models where the scalar couples to dark matter only is certainly well motivated. Future studies must assess how well these models can fit probes of the expansion history such as the supernova luminosity distance as well as linear probes such as the Cosmic Microwave Background. It would also be interesting to study the cosmology of more realistic models that include components neglected here such as radiation.

\section{ACKNOWLEDGMENTS}

I am incredibly grateful to Claes Uggla for several enlightening conversations and for bringing new techniques such as the center manifold to my attention. The final version of this work benefited greatly from discussions with Ruth Gregory and Kazuya Koyama. I would also like to thank Tomi Koivisto and Antonio Padilla for some interesting discussions. Finally, I am grateful to Carsten van de Bruck and Nelson Nunes for pointing out some typographical errors in the final version. Research at Perimeter Institute is supported by the Government of Canada through Industry Canada and by the Province of Ontario through the Ministry of Economic Development \& Innovation.

\section{APPENDIX A: DYNAMICAL SYSTEMS}

In this section we briefly review the aspects of dynamical systems theory required to study the disformal system.

\section{Fixed points and stability}

Consider a system described by $n$ first-order ordinary differential equations for $n$ variables $X_{i}$ as a function of some "time" coordinate $t$ and let a dot denote derivatives with respect to this. If the system can be written in the form

$$
\frac{\mathrm{d} X_{i}}{\mathrm{~d} t}=f_{i}\left(\left\{X_{j}\right\}\right)
$$

then it is known as an autonomous system and one can use dynamical systems techniques to classify the solutions.
The phase space of the system is the $n$-dimensional space spanned by $\left\{X_{j}\right\}$ and solutions of the system correspond to trajectories in this space. A fixed point of the system is one where $f_{i}=0 \forall i$. This is a set of $n$ algebraic equations that can be solved for the values of the fixed points $\left\{X_{j}^{c}\right\}$. If one tunes the variables to $\left\{X_{j}^{c}\right\}$ then the system will not evolve but one is generally interested in the behavior of arbitrary trajectories. In particular, if the fixed points are such that trajectories flow towards them at late times then the final state of the system is known independently of the initial conditions and so one can make important inferences about the late-time behavior of all solutions. In this case, the point is known as an attractor. If the trajectories flow away from the fixed point it is known as a repellor. Finding and classifying all of the fixed points of a system is tantamount to understanding the behavior of all possible solutions, which is especially important if one lacks analytic solutions. Cosmologically, one can calculate several important quantities such as the density parameter and the equation of state and so dynamical systems techniques have become an important tool to assess the viability of dark energy models.

One may determine the stability of the fixed points as follows. Consider linearizing the system about a fixed point such that $X_{i}=X_{i}^{\mathrm{c}}+\delta X_{i} . \delta X_{i}$ satisfies the equation

$$
\dot{\delta} \dot{X}_{i}=M_{i j} \delta X_{i}
$$

where

$$
M_{i j}=\frac{\partial f_{i}}{\partial X_{j}}
$$

and we have ignored second-order contributions since $\delta X_{i}$ is a small perturbation. This means that the stability analysis holds for regions of phase space close enough to the fixed points such that this linearization is a good approximation. Changing to the eigenbasis $\vec{e}_{i}$ of $M$ one has $\left(A_{j}\right.$ are new variables parametrizing the system in the eigenbasis)

$$
X_{i}=\sum_{j} A_{j}\left(\vec{e}_{j}\right)_{i}
$$

so that the equation for $\delta A_{j}$ is

$$
\dot{\delta A_{j}}=e_{j} \delta A_{j}
$$

where $e_{j}$ is the eigenvalue associated with $\vec{e}_{j}$. One then has $\delta A_{j} \sim e^{e_{j} t}$ and so the stability of the fixed point in the direction $\vec{e}_{j}$ is determined by $e_{j}$. There are several possibilities.

(i) $e_{j}$ is real and $e_{j}>0 \forall j$ : Trajectories in the direction flow away from the fixed point and it is known as an unstable node. 
(ii) $e_{j}$ is real and $e_{j}<0 \forall j$ : Trajectories in the direction flow towards the fixed point and it is known as a stable node.

(iii) $e_{j}$ is complex and $\Re e_{j}>0 \forall j$ : Trajectories spiral away from the fixed point. In this case the point is known as an unstable spiral.

(iv) $e_{j}$ is complex and $\Re e_{j}<0 \forall j$ : Trajectories spiral towards the fixed point; the point is known as a stable spiral in this case.

(v) There are a mixture of eigenvalues with differing signs for $\Re e_{j}$. In this case there are some stable directions and some unstable directions and the fixed point is known as a saddle point.

At late times, trajectories in phase space flow away from unstable nodes and towards stable nodes. The system may spend prolonged periods of time near saddle points but will ultimately evolve towards a stable node. We have not discussed the case where the system contains one or more zero eigenvalues. The simplest cosmological systems all have nonzero eigenvalues but, as we have seen above, disformal theories contain a fixed point with two zero directions and so here we must use more advanced techniques. This is the subject of the next subsection.

\section{Center manifolds}

The center manifold technique is used when the fixed point has one or more zero eigenvalues. Here we give a brief introduction and include only those aspects of the technique relevant for the problems studied in the main text.

Let us consider a fixed point described by $n-p$ negative (or negative real part) eigenvalues with corresponding eigenvectors $\vec{E}_{j}$ and $p$ zero eigenvalues with eigenvectors $\vec{e}_{j}$ [62]. One may expand the variables in terms of the eigenbasis such that

$$
X_{i}=\sum_{j=1}^{n-p} A_{j}^{s}\left(\vec{E}_{j}\right)_{i}+\sum_{j=n-p+1}^{n} A_{j}^{c}\left(\vec{e}_{j}\right)_{i},
$$

where we label stable directions as $A_{j}^{s}$ and zero eigenvalue or center directions as $A_{j}^{c}$. The zero eigenvalues indicate that a linear analysis is not sufficient to analyze the stability in the directions of $\vec{e}_{j}$. This is a problem with two time scales. Trajectories in the directions of $\vec{E}_{j}$ will converge to the attractor swiftly but the behavior of the components in the directions of $\vec{e}_{j}$ is unknown. The center manifold technique allows one to solve for the late-time behavior of $A_{j}^{c}$ by working on time scales such that $A_{j}^{s}$ have converged to their trajectories along the attractor. One then reduces the dimension of the phase space from $n$ to $p$ and can formulate the system as an autonomous one in the hope of being able to derive the behavior of $A_{j}^{c}$. This works as follows: the full $n$-dimensional system can be described by the autonomous system

$$
\begin{aligned}
& \frac{\mathrm{d} A_{i}^{s}}{\mathrm{~d} t}=g_{i}\left(\vec{A}^{s}, \vec{A}^{c}\right) i=1, \ldots, n-p \quad \text { and } \\
& \frac{\mathrm{d} A_{i}^{c}}{\mathrm{~d} t}=h_{i}\left(\vec{A}^{s}, \vec{A}^{c}\right) i=n-p+1, \ldots, n .
\end{aligned}
$$

Setting $g_{i}=0$ gives $n-p$ algebraic equations, which allows one to solve for $\vec{A}^{s}\left(\vec{A}^{c}\right)$ along trajectories at sufficiently late times such that the attractor has been reached in the $\vec{E}_{j}$ directions. The dynamics in the reduced $p$-dimensional phase space are then described by

$$
\frac{\mathrm{d} A_{i}^{c}}{\mathrm{~d} t}=h_{i}\left(\vec{A}^{s}\left(\vec{A}^{c}\right), \vec{A}^{c}\right) i=n-p+1, \ldots, n .
$$

This represents a new autonomous system that can be investigated using the techniques of Appendix A to discern the late-time behavior.

\section{APPENDIX B: EIGENVALUES AT THE FIXED POINTS}

In this appendix we list the eigenvalues for each fixed point. Points (18)-(20) are those of the two-dimensional phase space when $\gamma=\lambda / 2$ and so there are only two eigenvalues.

\section{Eigenvalues}

(1) $e_{1}=\alpha^{2}-3 / 2, e_{2}=-\alpha(\alpha+2 \gamma)-3 / 2, e_{3}=\alpha(\alpha+\lambda)+3 / 2$

(2) $e_{1}=3-\sqrt{6} \alpha, e_{2}=-\sqrt{6} \gamma-3, e_{3}=\sqrt{3 / 2} \lambda+3$

(3) $e_{1}=\sqrt{6} \alpha+3, e_{2}=\sqrt{6} \gamma-3, e_{3}=3-\sqrt{3 / 2} \lambda$

(4) $e_{1}=\lambda(\gamma-\lambda / 2), 1 / 2\left(\lambda^{2}-6\right), e_{3}=\lambda(\alpha+\lambda)-3$

(5) $e_{1}=3(2 \gamma-\lambda) / 2(\alpha+\lambda), \quad e_{2}=-\frac{\sqrt{3} \sqrt{-(\alpha+\lambda)^{2}\left(16 \alpha^{3} \lambda+4 \alpha^{2}\left(8 \lambda^{2}-15\right)+4 \alpha \lambda\left(4 \lambda^{2}-9\right)+21 \lambda^{2}-72\right)}+3(\alpha+\lambda)(2 \alpha+\lambda)}{4(\alpha+\lambda)^{2}}$, $e_{3}=\frac{\sqrt{3} \sqrt{-(\alpha+\lambda)^{2}\left(16 \alpha^{3} \lambda+4 \alpha^{2}\left(8 \lambda^{2}-15\right)+4 \alpha \lambda\left(4 \lambda^{2}-9\right)+21 \lambda^{2}-72\right)}-3(\alpha+\lambda)(2 \alpha+\lambda)}{4(\alpha+\lambda)^{2}}$ 
(6) $e_{1}=-\frac{1}{2}\left(\sqrt{4 \gamma^{2}-6}-2 \gamma\right)(2 \gamma-\lambda)$,

$$
\begin{aligned}
& e_{2}=\frac{1}{2}\left(3\left(\sqrt{\frac{8 \alpha^{2}-4 \alpha\left(\sqrt{4 \gamma^{2}-6}-4 \gamma\right)+10 \gamma^{2}-4 \gamma \sqrt{4 \gamma^{2}-6}-3}{2 \gamma\left(\sqrt{4 \gamma^{2}-6}+2 \gamma\right)-3}}-3\right)-\left(\sqrt{4 \gamma^{2}-6}-2 \gamma\right)(2 \alpha+5 \gamma)\right), \\
& e_{3}=-\frac{1}{2}\left(\left(\sqrt{4 \gamma^{2}-6}-2 \gamma\right)(2 \alpha+5 \gamma)+3\left(\sqrt{\frac{8 \alpha^{2}-4 \alpha\left(\sqrt{4 \gamma^{2}-6}-4 \gamma\right)+10 \gamma^{2}-4 \gamma \sqrt{4 \gamma^{2}-6}-3}{2 \gamma\left(\sqrt{4 \gamma^{2}-6}+2 \gamma\right)-3}}+3\right)\right)
\end{aligned}
$$

(7) $e_{1}=\frac{1}{2}\left(\sqrt{4 \gamma^{2}-6}+2 \gamma\right)(2 \gamma-\lambda), e_{2}=\frac{3\left(\left(\sqrt{4 \gamma^{2}-6}-2 \gamma\right)(2 \alpha-\gamma)+U_{1}-9\right)}{4 \gamma\left(\sqrt{4 \gamma^{2}-6}-2 \gamma\right)+6}$,

$e_{3}=-3 \frac{U_{2}}{2\left(\gamma\left(\sqrt{4 \gamma^{2}-6}-2 \gamma\right)+3\right)^{3}}+\frac{3\left(2 \alpha\left(2 \gamma^{2}-3\right)\left(\sqrt{4 \gamma^{2}-6}-2 \gamma\right)+4 \gamma^{4}-24 \gamma^{2}+3 \sqrt{4 \gamma^{2}-6} \gamma-2 \sqrt{4 \gamma^{2}-6} \gamma^{3}+27\right)}{2\left(\gamma\left(\sqrt{4 \gamma^{2}-6}-2 \gamma\right)+3\right)^{2}}$

(8) $e_{1}=\sqrt{6} \gamma+3, e_{2}=-\sqrt{6} \alpha+\sqrt{6} \gamma+6, e_{3}=\sqrt{\frac{3}{2}} \lambda+3$

(9) $e_{1}=3 / 2, e_{2}=3 / 2, e_{3}=3 / 2$

(10) $e_{1}=3-\sqrt{6} \gamma, e_{2}=\sqrt{6} \alpha-\sqrt{6} \gamma+6, e_{3}=3-\sqrt{\frac{3}{2}} \lambda$

(11) $e_{1}=-3, e_{2}=0, e_{3}=0$

(12) $e_{1}=\frac{1}{9}\left(\left(\sqrt{4(\alpha-\gamma)^{2}-18}+2 \alpha-2 \gamma\right)(\alpha-\gamma)-18\right)$, $e_{2}=\frac{1}{9}\left((\alpha+2 \gamma)\left(\sqrt{4(\alpha-\gamma)^{2}-18}+2 \alpha-2 \gamma\right)+9\right)$,

$e_{3}=\frac{1}{18}\left(\left(\sqrt{4(\alpha-\gamma)^{2}-18}+2 \alpha-2 \gamma\right)(2 \alpha-2 \gamma+3 \lambda)+18\right)$

(13) $e_{1}=\frac{1}{9}\left(\left(-\sqrt{4(\alpha-\gamma)^{2}-18}+2 \alpha-2 \gamma\right)(\alpha-\gamma)-18\right)$ $e_{2}=\frac{1}{9}\left(9-(\alpha+2 \gamma)\left(\sqrt{4(\alpha-\gamma)^{2}-18}-2 \alpha+2 \gamma\right)\right)$,

$e_{3}=\frac{1}{18}\left(18-\left(\sqrt{4(\alpha-\gamma)^{2}-18}-2 \alpha+2 \gamma\right)(2 \alpha-2 \gamma+3 \lambda)\right)$

(14) $e_{1}=\frac{1}{2} \lambda(\lambda-2 \gamma), e_{2}=\frac{1}{2}\left(\lambda^{2}-6\right), e_{3}=\alpha \lambda-\gamma \lambda+\frac{3 \lambda^{2}}{2}-3$

(15) $e_{1}=\frac{3 \lambda-6 \gamma}{2 \alpha-2 \gamma+3 \lambda}, \quad e_{2}=-\frac{3\left(U_{3}+(\alpha-\gamma+\lambda)(\lambda(2 \alpha-2 \gamma+3 \lambda)-12)(2 \alpha-2 \gamma+3 \lambda)^{5 / 2}\right)}{(2 \alpha-2 \gamma+3 \lambda)^{7 / 2}(\lambda(2 \alpha-2 \gamma+3 \lambda)-12)}, \quad e_{3}=\frac{3\left(U_{3}-(\alpha-\gamma+\lambda)(2 \alpha-2 \gamma+3 \lambda)^{5 / 2}(\lambda(2 \alpha-2 \gamma+3 \lambda)-12)\right)}{(2 \alpha-2 \gamma+3 \lambda)^{7 / 2}(\lambda(2 \alpha-2 \gamma+3 \lambda)-12)}$

(16) $e_{1}=\frac{1}{2}\left(\sqrt{4 \gamma^{2}-6}+2 \gamma\right)(2 \gamma-\lambda), \quad e_{2}=\frac{1}{2}\left(\left(\sqrt{4 \gamma^{2}-6}+2 \gamma\right)(2 \alpha+5 \gamma)+U_{3}-9\right)$,

$$
e_{3}=\frac{1}{2}\left(\left(\sqrt{4 \gamma^{2}-6}+2 \gamma\right)(2 \alpha+5 \gamma)-U_{3}-9\right)
$$

(17) $e_{1}=-\frac{1}{2}\left(\sqrt{4 \gamma^{2}-6}-2 \gamma\right)(2 \gamma-\lambda), \quad e_{2}=\frac{1}{2}\left(\left(\sqrt{4 \gamma^{2}-6}+2 \gamma\right)(2 \alpha+5 \gamma)+U_{4}-9\right)$,

$$
e_{3}=\frac{1}{2}\left(\left(\sqrt{4 \gamma^{2}-6}+2 \gamma\right)(2 \alpha+5 \gamma)-U_{4}-9\right)
$$

(18) $e_{1}=\frac{1}{2}\left(\lambda^{2}-6\right), e_{2}=\lambda(\alpha+\lambda)-3$

(19) $e_{1}=-\frac{6\left(2 \alpha^{2} \lambda+3 \alpha\left(\lambda^{2}-4\right)+\lambda^{3}\right)+\sqrt{3} U_{4}+3 \tilde{\Lambda}^{2}(2 \alpha+\lambda)(2 \alpha(\alpha+\lambda)+3)-36 \lambda}{4(\alpha+\lambda)\left(2(\alpha+\lambda)\left(\alpha \tilde{\Lambda}^{2}+\lambda\right)+3\left(\tilde{\Lambda}^{2}-4\right)\right)}, \quad e_{2}=\frac{-6\left(2 \alpha^{2} \lambda+3 \alpha\left(\lambda^{2}-4\right)+\lambda^{3}\right)+\sqrt{3} U_{4}-3 \tilde{\Lambda}^{2}(2 \alpha+\lambda)(2 \alpha(\alpha+\lambda)+3)+36 \lambda}{4(\alpha+\lambda)\left(2(\alpha+\lambda)\left(\alpha \tilde{\Lambda}^{2}+\lambda\right)+3\left(\tilde{\Lambda}^{2}-4\right)\right)}$

(20) $e_{1}=\frac{\left(\Lambda^{2}-2\right)\left(\Lambda^{2}\left(4 \alpha \lambda+5 \lambda^{2}-18\right)-\Lambda(4 \alpha+5 \lambda) \sqrt{\left(\lambda^{2}-6\right) \Lambda^{2}+12}+36\right)-\sqrt{2} U_{6}}{4\left(\Lambda^{2}-2\right)}, \quad e_{2}=\frac{\left(\Lambda^{2}-2\right)\left(\Lambda^{2}\left(4 \alpha \lambda+5 \lambda^{2}-18\right)-\Lambda(4 \alpha+5 \lambda) \sqrt{\left(\lambda^{2}-6\right) \Lambda^{2}+12}+36\right)+\sqrt{2} U_{6}}{4\left(\Lambda^{2}-2\right)}$ where

$U_{1}^{2}=9-8 \alpha^{2}\left(2 \gamma\left(\sqrt{4 \gamma^{2}-6}-2 \gamma\right)+3\right)-4 \alpha\left(4\left(\sqrt{4 \gamma^{2}-6}-2 \gamma\right) \gamma^{2}+3 \sqrt{4 \gamma^{2}-6}\right)+8 \gamma^{4}+6 \gamma^{2}-6 \sqrt{4 \gamma^{2}-6} \gamma-4 \sqrt{4 \gamma^{2}-6} \gamma^{3}$,

$$
\begin{aligned}
U_{2}^{2}= & -\left(2 \gamma^{2}-3\right)^{3}\left(8 \alpha^{2}\left(4 \gamma\left(4 \gamma\left(\gamma\left(\sqrt{4 \gamma^{2}-6}-2 \gamma\right)+3\right)-3 \sqrt{4 \gamma^{2}-6}\right)-9\right)\right. \\
& \left.+4 \alpha\left(4 \gamma\left(4\left(2 \gamma\left(\sqrt{4 \gamma^{2}-6}-2 \gamma\right)+3\right) \gamma^{2}+9\right)-9 \sqrt{4 \gamma^{2}-6}\right)-64 \gamma^{6}+54 \gamma^{2}+32 \sqrt{4 \gamma^{2}-6} \gamma^{5}+24 \sqrt{4 \gamma^{2}-6 \gamma^{3}}+27\right),
\end{aligned}
$$




$$
\begin{aligned}
U_{3}^{2}= & (2 \alpha-2 \gamma+3 \lambda)^{5}\left(38 \lambda^{3}(\alpha-\gamma)+\lambda^{2}\left(35(\alpha-\gamma)^{2}-12\right)+10 \lambda\left((\alpha-\gamma)^{2}-6\right)(\alpha-\gamma)\right. \\
& \left.-36\left((\alpha-\gamma)^{2}+1\right)+12 \lambda^{4}\right)(\lambda(2 \alpha-2 \gamma+3 \lambda)-12), \\
U_{4}^{2}= & \left(\left(\sqrt{4 \gamma^{2}-6}+2 \gamma\right)(2 \alpha+5 \gamma)-9\right)^{2}-8\left(\alpha\left(4 \gamma\left(\gamma\left(\sqrt{4 \gamma^{2}-6}+2 \gamma\right)-3\right)-3 \sqrt{4 \gamma^{2}-6}\right)\right. \\
& \left.+\gamma\left(2 \gamma\left(4 \gamma\left(\sqrt{4 \gamma^{2}-6}+2 \gamma\right)-15\right)-9 \sqrt{4 \gamma^{2}-6}\right)+9\right), \\
U_{5}^{2}= & -\left(\tilde{\Lambda}^{2}(2 \alpha(\alpha+\lambda)+3)\left(16 \alpha^{3} \lambda+4 \alpha^{2}\left(8 \lambda^{2}-15\right)+4 \alpha \lambda\left(4 \lambda^{2}-9\right)+21 \lambda^{2}-72\right)\right. \\
& \left.-6\left(20 \alpha^{3} \lambda+8 \alpha^{2}\left(5 \lambda^{2}-9\right)+3 \alpha\left(7 \lambda^{2}-16\right) \lambda+\lambda^{4}+18 \lambda^{2}-72\right)\right)\left(2(\alpha+\lambda)\left(\alpha \tilde{\Lambda}^{2}+\lambda\right)+3\left(\tilde{\Lambda}^{2}-4\right)\right) \quad \text { and } \\
U_{6}^{2}= & \Lambda^{4}\left((4 \alpha+3 \lambda)\left(4 \alpha\left(\lambda^{2}-3\right)+3 \lambda\left(\lambda^{2}-5\right)\right)+18\right)-12 \Lambda(4 \alpha+3 \lambda) \sqrt{\left(\lambda^{2}-6\right) \Lambda^{2}+12} \\
& -\Lambda^{3}(4 \alpha+3 \lambda)\left(4 \alpha \lambda+3 \lambda^{2}-6\right) \sqrt{\left(\lambda^{2}-6\right) \Lambda^{2}+12}+6 \Lambda^{2}((4 \alpha+3 \lambda)(4 \alpha+5 \lambda)-12)+72 .
\end{aligned}
$$

\section{APPENDIX C: THE $x$ - $Y$-W SYSTEM}

Here, we present the autonomous system written using the variables $Y$ and $W$ defined in (46) and (47):

$$
\begin{aligned}
\frac{\mathrm{d} x}{\mathrm{~d} N}= & \frac{\sqrt{6}\left(x^{2}+Y(Y+2)\right)\left((W+1)\left(2 \alpha+(W+1)\left(-\alpha+3 x(2 x(\alpha-\gamma)+\sqrt{6})-3 \lambda(Y+1)^{2}\right)\right)-\alpha\right)}{2(W+1)\left((W+1)\left(9 x^{2}+3(Y+1)^{2}-4\right)+2\right)-2} \\
& +\frac{3}{2} x\left(x^{2}-Y(Y+2)\right)-3 x+\sqrt{6} \lambda(Y+1)^{2}, \\
\frac{\mathrm{d} Y}{\mathrm{~d} N}= & -\frac{1}{2}(Y+1)\left(-3 x^{2}+\sqrt{6} \lambda x+3 Y(Y+2)\right) \text { and } \\
\frac{\mathrm{d} W}{\mathrm{~d} N}= & \frac{1}{2} W(W+1)\left(3 x^{2}-2 \sqrt{6} \gamma x-3 Y(Y+2)\right) .
\end{aligned}
$$

[1] A. G. Riess et al. (Supernova Search Team), Astron. J. 116, 1009 (1998).

[2] S. Perlmutter et al. (Supernova Cosmology Project), Astrophys. J. 517, 565 (1999).

[3] T. Clifton, P. G. Ferreira, A. Padilla, and C. Skordis, Phys. Rep. 513, 1 (2012).

[4] A. Joyce, B. Jain, J. Khoury, and M. Trodden, arXiv:1407.0059.

[5] J. Khoury and A. Weltman, Phys. Rev. Lett. 93, 171104 (2004).

[6] J. Khoury and A. Weltman, Phys. Rev. D 69, 044026 (2004).

[7] K. Hinterbichler, J. Khoury, A. Levy, and A. Matas, arXiv:1107.2112.

[8] P. Brax, C. van de Bruck, A.-C. Davis, and D. Shaw, Phys. Rev. D 82, 063519 (2010).

[9] A. Vainshtein, Phys. Lett. 39B, 393 (1972).

[10] Note that some theories such as massive gravity [11] utilize these mechanisms, although the coupling to matter is only seen explicitly in the decoupling limit.
[11] C. de Rham, G. Gabadadze, and A. J. Tolley, Phys. Rev. Lett. 106, 231101 (2011).

[12] J.D. Bekenstein, in Marcel Grossmann Meeting on General Relativity, edited by F. Satō and T. Nakamura (World Scientific, Singapore, 1992), p. 905.

[13] J. D. Bekenstein, Phys. Rev. D 48, 3641 (1993).

[14] T. S. Koivisto, arXiv:0811.1957.

[15] M. Zumalacarregui, T. Koivisto, D. Mota, and P. RuizLapuente, J. Cosmol. Astropart. Phys. 05 (2010) 038.

[16] J. Noller, J. Cosmol. Astropart. Phys. 07 (2012) 013.

[17] M. Zumalacarregui, T. S. Koivisto, and D. F. Mota, Phys. Rev. D 87, 083010 (2013).

[18] M. Zumalacrregui and J. Garca-Bellido, Phys. Rev. D 89, 064046 (2014).

[19] T. Koivisto, D. Wills, and I. Zavala, arXiv:1312.2597.

[20] T. S. Koivisto and F. R. Urban, arXiv:1407.3445.

[21] J. Sakstein, J. Cosmol. Astropart. Phys. 12 (2014) 012.

[22] N. Kaloper, Phys. Lett. B 583, 1 (2004). 
[23] A.-C. Davis, E. A. Lim, J. Sakstein, and D. Shaw, Phys. Rev. D 85, 123006 (2012).

[24] B. Jain, V. Vikram, and J. Sakstein, Astrophys. J. 779, 39 (2013).

[25] P. Brax, A.-C. Davis, and J. Sakstein, arXiv:1301.5587.

[26] J. Sakstein, Phys. Rev. D 88, 124013 (2013).

[27] V. Vikram, J. Sakstein, C. Davis, and A. Neil, arXiv:1407.6044.

[28] J. Sakstein, B. Jain, and V. Vikram, arXiv:1409.3708.

[29] P. C. Freire, N. Wex, G. Esposito-Farese, J. P. Verbiest, M. Bailes, B. A. Jacoby, M. Kramer, I. H. Stairs, J. Antoniadis, and G. H. Janssen, Mon. Not. R. Astron. Soc. 423, 3328 (2012).

[30] J. Khoury, arXiv:1409.0012.

[31] One could screen this using the Damour-Polyakov effect [32], although this has not yet been investigated.

[32] T. Damour and A. M. Polyakov, Nucl. Phys. B423, 532 (1994).

[33] E. J. Copeland, A. R. Liddle, and D. Wands, Phys. Rev. D 57, 4686 (1998).

[34] D. J. Holden and D. Wands, Phys. Rev. D 61, 043506 (2000).

[35] B. Gumjudpai, T. Naskar, M. Sami, and S. Tsujikawa, J. Cosmol. Astropart. Phys. 06 (2005) 007.

[36] E. J. Copeland, M. Sami, and S. Tsujikawa, Int. J. Mod. Phys. D 15, 1753 (2006).

[37] G. Hinshaw et al. (WMAP), Astrophys. J. Suppl. Ser. 208, 19 (2013).

[38] Note that we are using the conventions of [21]. A dictionary to convert these conventions to others in the literature such as [17] can be found there.

[39] It is the Jordan frame energy-momentum tensor that is conserved since matter is minimally coupled in this frame.

[40] It is assumed that $\Phi \sim \Psi \sim \varphi \sim \Phi_{\mathrm{N}}$, where $\Phi_{\mathrm{N}}$ is the Newtonian potential, so that terms such as $\dot{\varphi}^{2} \sim \Phi_{\mathrm{N}} \nabla^{2} \Phi_{\mathrm{N}}$ can be neglected. This ansatz can be checked selfconsistently (see the discussion in [21]) and allows one to systematically construct the nonrelativistic limit.

[41] Note that this assumes $\dot{\phi}_{\infty} \ll \Lambda$. If this is not the case then there are time-dependent corrections to Newton's constant (see the discussion below).

[42] One could perform the same analysis for the general model but this is cumbersome and does not add anymore insight. The pathologies discussed here arise due to the disformal coupling and so it suffices to consider the simplest case for the purposes of elucidation.

[43] E. Bellini and I. Sawicki, J. Cosmol. Astropart. Phys. 07 (2014) 050.

[44] C. van de Bruck and G. Sculthorpe, Phys. Rev. D 87, 044004 (2013).

[45] P. Brax, C. Burrage, A.-C. Davis, and G. Gubitosi, J. Cosmol. Astropart. Phys. 11 (2013) 001.

[46] C. van de Bruck, J. Morrice, and S. Vu, Phys. Rev. Lett. 111, 161302 (2013).

[47] D. Bettoni and S. Liberati, Phys. Rev. D 88, 084020 (2013).

[48] P. Brax, arXiv:1211.5237.

[49] A. Barreira, B. Li, C. Baugh, and S. Pascoli, arXiv:1308.3699.

[50] This is because the analysis employed below uses $\ln a(t)$ as a time coordinate and eliminates $H(a)$ through the Friedmann constraint. This choice implicitly assumes that $a(t)$ is monotonically increasing and can hence be used as a time coordinate. One could instead work with a larger phase space in order to treat $H$ as a dynamical variable and capture any deviations from monotonicity but it is simpler to work in the Jordan frame. This exercise is postponed for follow-up work.

[51] One may wonder whether reintroducing $A$ and $B$ can change this argument. One can always perform a field redefinition to remove the function multiplying the disformal factor and hence any effects of having $B \neq 1$ can be equivalently thought of as arising due to noncanonical kinetic terms for the scalar. The conformal factor is less subtle but in this case any acceleration in the Jordan frame is due to conformal effects only and hence this scenario is not relevant for this work.

[52] Note that this was studied briefly in [17], Appendix C.

[53] By this, we mean that at any fixed time the fixed points found here will be solutions of the equations. Whether or not they are reached however depends on the specific model and in general one expects new fixed points and that the properties found here may be destroyed. In practice, models where the dynamics are predictable-for example potentials with minima so that we expect $\lambda(\phi) \rightarrow 0$ at late times-tend to approach these fixed points but we stress that a more complicated analysis is required to draw any definite conclusions. This is especially true in cases where either the potential or the disformal factor can become zero.

[54] The parameter space is spanned by $\gamma, \alpha, \lambda$ and $\Lambda / m_{0}$.

[55] One may solve for $\phi$ from the definition of $y$ to find $B=(\sqrt{3} H y)^{2 \gamma / \lambda}$. When $\gamma=\lambda / 2$ one can scale $H$ out of the equations without the need to define a new quantity $z$ whereas when this is not the case a new variable is needed. Therefore, one may think of this tuning as an enhanced symmetry of the equations whereby one can scale $H, V$ and $\dot{\phi}_{\infty}$ to obtain new solutions of the system without the need to scale $B$.

[56] L. Amendola, Phys. Rev. D 62, 043511 (2000).

[57] Note that $z=0$ does not necessarily mean that $B=0$. Since $z \propto H$ these points may correspond to the infinite future where $H=0$.

[58] We have not included fixed points where $Z>1$ or $Z<0$, which lie outside the physical state space. Furthermore, one finds that there is an additional fixed point when $\gamma \rightarrow \infty$ where $Z=1$, which we also ignore. This may be relevant for models where $\gamma$ can reach infinity.

[59] A. Alho and C. Uggla, arXiv:1406.0438.

[60] One should note that we refer to this phenomena as pathology resistance in order to make contact with previous works. We consider models exhibiting metric singularities to be unviable for the reasons discussed in Sec. II A. One should note that it is possible to construct theories where the visible sector coupled to the Einstein frame metric but dark matter couples to the Jordan frame metric. In this case, it is less clear whether these solutions are indeed pathological since the nonrelativistic limit is well defined for visible matter and fifth forces are not an issue.

[61] Note that this data set assumes that $\omega$ is fixed whereas it varies in our model and so a more realistic method would be to use a varying $\omega$ fit such as the $\omega_{0}-\omega_{a}$ parametrization. The analysis here is a proof of principle only and so we will not concern ourselves with a more realistic data analysis.

[62] We are interested in stable fixed points since these describe the late-time behavior of the system and so we will not consider positive eigenvalues here. 